\title{
老化认知神经科学: 研究现状与未来展望
}

\author{
刘涵慧, 李会杰, ${ }^{2,3}$ \\ 1. 中国青年政治学院青少年工作系, 北京 100089 ; \\ 2. 中国科学院心理研究所行为科学重点实验室, 北京 100101; \\ 3. 中国科学院大学心理学系, 北京 100049 \\ *联系人, E-mail: lihj@psych.ac.cn
}

收稿日期: 2020-08-04; 接受日期: 2020-10-20; 网络版发表日期: 2021-01-21

国家自然科学基金(批准号: 31871143)和中国科学技术协会学科发展项目(批准号: 2018XKFZ03)资助

\begin{abstract}
摘要人口老龄化已经成为我国乃至全球面临的一个严峻的社会问题. 开展老化认知神经科学研究, 从认知、脑 结构和脑功能层面揭示脑老化的机制和规律，进而探索并开发出延缓认知下降的方法和工具，对保持老年脑健康 及提升生活质量具有重要意义. 本文从脑结构老化、脑功能老化以及脑血流量与脑老化等方面概述了认知老化 的神经机制. 前人从补偿和去分化角度构建了多个认知老化理论和假说, 然而, 这些理论和假说较少考虑到脑结 构老化、静息态研究及脑血流量的研究发现. 坚持规律的运动、多做一些认知参与的活动、保持健康的生活方 式和饮食模式, 有助于老年人保持认知功能并延缓认知与脑老化. 在老化认知神经科学未来发展方向上, 从认知 老化理论的发展以及成人毕生发展视角，对脑老化的脑功能网络研究、脑老化与可塑性以及基因对脑老化的影 响等方面进行了展望。最后, 本文从建立中国成人毕生发展行为-基因-脑数据库、使用和研发新技术研究认知老 化、深入探索有效的脑功能改善方法、开展农村老年人研究及临床老年群体研究等方面, 对国内老化认知神经 科学的发展趋势进行了展望.
\end{abstract}

关键词 老年人, 老化, 前额叶, 干预, 神经科学

随着科技的进步和医疗卫生水平的提高，世界人 口的平均寿命不断增长，人口老龄化已经成为我国乃 至全球面临的一个严峻的社会问题. 联合国经济和社 会事务部发布的数据表明，全球 65 岁以上的老年人在 2019年达到7.03亿，2050年将达到15亿 亿 $^{[1]}$. 从国家统计 局发布的数据来看, 截至 2019 年底, 我国大陆60周岁及 以上的老年人约 2.54 亿，占总人口的 $18.1 \% ， 65$ 周岁及 以上的老年人约 1.76 亿，占总人口的 $12.6 \%^{[2]}$. 人口老 龄化不仅给社会和家庭带来经济、养老、医护等负
担, 更因增龄性衰老直接降低老年人的生活质量. 随着 年龄的增长，老年人在加工速度、视空间加工、注 意、情节记忆、执行功能、空间导航等认知功能上会 出现不同程度的下降 ${ }^{[3 \sim 10]}$. 认知能力下降会导致老年 人工具性日常生活能力困难以及独立生活能力丧 失 $^{[11]}$.

开展老化认知神经科学研究, 从认知、脑结构和 脑功能层面揭示脑老化的机制，探索并开发出延缓脑 老化的干预方法，对保持老年脑健康及提升老年人生

引用格式: 刘涵慧, 李会杰. 老化认知神经科学: 研究现状与未来展望. 中国科学: 生命科学, 2021, 51: 743-763

Liu H H, Li H J. Cognitive neuroscience of aging: present research status and future prospect (in Chinese). Sci Sin Vitae, 2021, 51: 743-763, doi: $10.1360 /$ SSV-2020-0256 
活质量具有重要意义。本文在对认知及脑老化相关研 究进行梳理的基础上, 结合国内外已有研究, 进行了 老化认知神经科学未来发展方向的预测和展望, 并提 出了我国老化认知神经科学未来发展的路线图.

在本文中, 除特殊指代外, 老年人均指认知健康的 老年人, 即未被诊断为轻度认知损伤(mild cognitive impairment, MCI)、阿尔茨海默病(Alzheimer's disease, $\mathrm{AD}$ )及其他以认知下降为主要临床表现的疾病 ${ }^{[12]}$. 认 知指获得知识和理解力涉及的心理过程 ${ }^{[13]}$. 不同研究 者对认知的分类有较大差异，一般包括感知觉、注意 和意识、语言、学习和记忆以及认知控制等 ${ }^{[14]}$.

\section{1 认知老化的神经机制}

人们在成年后认知能力随龄下降，这一现象被称 为认知老化 ${ }^{[15]}$. 除言语知识外, 20 92岁成年人的工作 记忆、短时记忆、长时记忆、加工速度和言语知识等 认知能力自 20 岁起便呈现随龄下降的趋势 ${ }^{[16]}$. 一项涵 盖18 89岁成年人的研究发现, 成年人在推理、视空 间加工、情节记忆、知觉速度以及词汇等领域的成绩 随增龄下降 ${ }^{[17]}$. 近期研究发现, 词汇、知识经验等会 随年龄的增加而增长，到70岁后会出现衰退; 记忆、 注意、推理等认知能力在成年早期达到峰值后便开始 呈现下降趋势, 进入老年阶段后, 衰退进程加快 ${ }^{[18]}$. 国 内学者发现, 加工速度、工作记忆、心算、空间表象 和记忆再认在16 19岁达到高峰, 在20岁以后出现缓 慢下降, 60岁后下降速度变快 ${ }^{[19]}$. 针对年龄跨度35 98 岁的横断研究发现，成年人在韦氏记忆量表和简易精 神状态量表上的得分随龄下降 ${ }^{[20]}$. 追踪研究也发现了 相同的随龄下降趋势. 柏林老化研究对132名老年人 进行了为期 6 年的追踪，发现加工速度、记忆和词语 流畅性随年龄增长而下降 ${ }^{[21]}$. 澳大利亚一项涵盖2087 名老年人的纵向研究发现, 听力、记忆、言语能力和 加工速度呈现显著的随龄下降 ${ }^{[22]}$. 针对6476名美国老 年人开展的为期 9 年的追踪研究也发现，老年人各项 认知功能均呈现出下降趋势 ${ }^{[23]}$. 尽管老年人在加工速 度、情节记忆、工作记忆、抑制等多项认知能力上表 现出下降，但情节记忆缺损被认为是老年人认知下降 最显著的特点和最敏感的指标 ${ }^{[24,25]}$. 情节记忆随龄下 降最多的任务是自由回忆 ${ }^{[26]}$ 、时间顺序记忆 ${ }^{[27]}$ 和来 源记忆 ${ }^{[28]}$.
目前有多个理论和假说来解释认知老化. 认知老 化研究者经常观察到老年人在行为和神经加工上反应 变慢, 据此提出了加工速度老化理论 ${ }^{[29,30]}$. 加工速度变 慢与白质纤维束的结构完整性 ${ }^{[31,32]}$ 和脑灰质体积下 降 ${ }^{[33]}$ 有关. 随着脑老化, 多巴胺水平下降, 神经噪音增 加, 神经信号的信噪比降低 ${ }^{[34-36]}$, 老年人需要更多的 时间对神经信号进行评估，因此完成认知任务的速度 变慢. 抑制缺损假说 ${ }^{[37,38]}$ 是另一个影响广泛的认知老 化假说. 该假说认为, 在阻止无关信息、删除不再需 要的无关信息以及保持优势反应上，老年人表现出抑 制控制下降. 老年人抑制控制能力下降，会导致工作 记忆、学习以及理解能力的降低 ${ }^{[39]}$. 老年人抑制控制 下降被认为是由前额叶结构和功能变化导致的, 这种 观点被称为前额叶功能老化理论 ${ }^{[40,41]}$.

老化认知神经科学的主要挑战是揭示认知能力随 增龄下降的脑机制. 近年来, 磁共振成像 (magnetic resonance imaging, MRI)技术在揭示认知下降的脑机制 中发挥了重要作用. 结构MRI、基于任务的功能磁共 振成像(functional MRI, fMRI)和静息态fMRI分别从脑 结构、脑激活和脑自发活动的层面探索脑老化的神经 机制, 得出了很多重要的发现 ${ }^{[42,43]}$.

\section{1 脑结构老化}

脑结构老化主要表现为灰质萎缩、白质减少以及 脑室扩张 ${ }^{[4]}$. 研究者考察了 465名17 79岁成年人的脑 灰质和白质的随龄变化，发现全脑灰质体积随龄线性 下降. 横向对比发现灰质年均萎缩率约 $0.2 \%$, 追踪研 究表明年均萎缩率为 $0.3 \%$ 0.5\%; 从局部脑区看, 双侧 脑岛、额上回、中央沟、扣带回等脑区呈现加速萎 缩，而杏仁核、海马和内嗅皮层则没有或呈现出很小 的随龄变化 ${ }^{[45]}$. 然而, 其他研究者则发现, 海马和小脑 随龄呈现快速的萎缩 ${ }^{[46]}$. 对207名23 87岁成年人开展 的研究发现，不管全脑还是外侧枕叶、顶下皮层、缘 上回、题中回、题上回、楔前叶、后压部皮层、后扣 带、海马旁回以及内嗅皮层，其灰质表面积、皮层厚 度和皮层体积均随龄下降，尽管不同脑区下降速率不

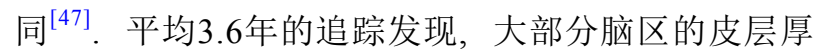
度、表面积和体积的萎缩率分别为 $0.35 \% 、 0.19 \%$ 和 $0.51 \%$. 在歌叶和顶叶脑区, 呈现出加速的萎缩, 前额叶 和前扣带萎缩速度相对较慢 ${ }^{[47]}$. 白质的老化遵循“先入 后出”的规律, 较早发育成熟的白质在老化过程中退化 
慢, 较晚发育成熟的白质则退化快 ${ }^{[48]}$. 在一项考察脑 白质纤维束纵向弛豫率的毕生发展(7 85 岁)研究中, 研究者发现, 纵向弛豫率呈现倒U型分布, 在中年期达 到顶峰. 这说明脑白质的成熟可能持续到中年, 随后开 始下降 ${ }^{[49]}$. 由于该研究只纳入了102名被试, 尚待更大 样本的研究加以证实.

脑结构的随龄变化对认知功能有直接的影响. 一 年的追踪研究表明, 左侧内嗅皮层的灰质萎缩可以预 测延迟言语记忆的下降. 基线测试时左侧题叶的白质 和灰质体积越小, 老年人的语义记忆会经历更大的下

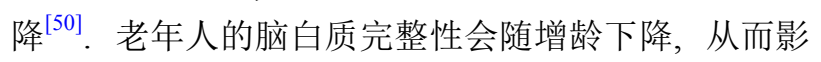
响加工速度和执行功能等认知能力 ${ }^{[51]}$. 对特定脑区而 言, 前额叶白质高信号和灰质萎缩与较差的执行功 能、工作记忆以及信息加工速度有关 ${ }^{[4]}$, 海马和内嗅 皮层体积的减少预示着记忆能力的下降 ${ }^{[52]}$. 此外, 脑 结构的变化对老年人日常生活功能也会产生影响. 行 动能力受损老年人的白质信号强度是未受损对照组老 年人的 5 倍, 白质信号强度增加的程度可以预测行动受 损恶化的程度 ${ }^{[53]}$.

\section{2 脑功能老化}

(1) 基于任务的脑功能老化研究. 研究者系统综述 了认知老化的任务fMRI研究, 他们把纳入的 80 篇研究 按照任务分成了知觉、记忆编码、记忆提取以及执行 功能等领域, 结果发现, 老年人的前额叶在不同任务领 域中都表现出了更强的激活, 而青年人则在枕叶区域 的激活强于老年人 ${ }^{[54]}$. 对于老化认知神经科学研究的 这种发现, 研究者从补偿角度提出了多个理论或假说 进行解释. 老化的前后转移模型(posterior-anterior shift in aging)认为, 老年人在执行认知任务时会激活更多 的前额叶等脑区以补偿后部枕叶脑区活动的降低, 如 面孔和位置知觉任务中, 老年人在颢枕区激活低于青 年人, 而前额叶激活高于青年人 ${ }^{[55]}$. 老年人在完成注 意任务、视知觉任务、视空间任务、工作记忆任务时 均存在脑激活的前后转移现象 ${ }^{[56-58]}$. 半球非对称性下 降假说(reduction of task-related hemispheric lateralization, HAROLD)认为, 老年人需要激活双侧前额叶皮层 来完成认知任务 (如情节记忆、再认、执行功能等), 而青年人则只需激活单侧前额叶皮层即可 ${ }^{[59]}$. 神经环 路的补偿相关利用假说 (compensation-related utilization of neural circuits hypothesis)认为, 老年人前额叶
补偿性的激活主要发生在低负荷认知任务中, 由于老 年人加工效率低, 其需调用更多的神经资源才能获得 和青年人相当的行为成绩; 对于较难的认知任务, 当 补偿作用达到上限时, 前额叶激活增加也无法起到有 效的补偿作用 ${ }^{[60]}$. 老化与认知的脚手架理论(The Scaffolding Theory of Aging and Cognition, STAC)认为, 老 年人激活更多的前额叶是为了保持较高的认知表现以 及补偿脑功能的下降. 脑结构和功能都可以预测认知 能力和认知老化的速率, 而补偿性的脚手架则可以调 节这种预测 ${ }^{[61,62]}$.

基于任务的脑功能老化研究中, 与青年人相比, 老年人在完成不同任务时会表现出相似的脑激活模 式. 换句话说, 老年人在完成不同任务时脑激活模式 的特异性下降, 研究者据此提出了去分化假说(dedifferentiation hypothesis $)^{[63 \sim 65]}$. 考察老年人和青年人在 执行不同类型视觉刺激(面孔和房子)的脑激活特点发 现, 老年人在加工两类视觉刺激时激活特异性降低, 并且这种降低可以预测老年人流体加工能力 (点比较 任务、数字表征、连线测试、言语流畅性等) 的下 降 ${ }^{[66]}$.

近年来, 研究者开始关注老年人执行认知任务时 的脑功能网络活动特点. 在自由回忆成绩降低的试次 中, 老年人额顶网络和扣带-盖部网络活动升高 ${ }^{[67]}$. 研 究者考察老年人和青年人执行伦敦塔任务时脑功能网 络活动发现, 在计划过程中, 两组被试在激活额顶控制 网络的同时均抑制了默认网络的活动 ${ }^{[68]}$. 另一项研究 发现, 老年人在视空间加工中对默认网络调节失败, 反映了老年人对网络交互的控制减弱及随任务需求动 态调节能力下降 ${ }^{[69]}$. 针对已发表的 114 项基于任务 fMRI的研究, 研究者发现在脑功能网络水平上, 与青 年人相比, 老年人额顶网络和默认网络激活升高, 视觉 网络激活降低 ${ }^{[70]}$. 通过大尺度功能网络和基于贝叶斯 似然估计相结合的方法, 研究者系统综述了 MCI和 $\mathrm{AD}$ 的任务fMRI研究, 结果发现与健康老年人相比, 随着 认知异常老化程度的进展, MCI和 $\mathrm{AD}$ 病人脑功能网络 下降呈现出不同的特点, 但是激活升高的功能网络中, 控制系统(额顶网络、背侧注意和腹侧注意网络)均占 据了超过 $50 \%$ 的比例, 这说明在完成认知任务时, MCI 和 $\mathrm{AD}$ 病人都激活了较多的控制系统, 提示控制系统在 认知异常老化过程中扮演着补偿等调控作用 ${ }^{[71]}$. 这些 研究结果表明, 脑功能网络特别是额顶网络在脑老化 
过程中可能发挥重要调控作用, 由于认知老化伴随着 重要的认知控制能力下降, 因此, 从额顶功能网络的 调控机制入手研究认知老化的脑机制具有重要意义.

（2）基于自发活动的脑功能老化研究. 当前, 在老 化认知神经科学研究中, 基于脑自发活动的静息态 fMRI研究为认识脑老化提供了新的视角. 基于 35 个中 心、1414名被试的静息态fMRI数据，研究者发现，老 年人默认网络的低频振幅以及功能连接均随增龄下 降 ${ }^{[72]}$. 老年人大尺度的脑网络拓扑效率下降，特别是 默认网络和背侧注意网络 ${ }^{[73]}$. 对913名被试的静息态 功能连接密度研究发现, 默认网络和背侧注意网络的 功能连接密度随龄下降, 而躯体感觉和小脑网络则随 龄增长, 长距离比短距离功能连接密度下降更快, 对 于老化效应更敏感 ${ }^{[74]}$. 该研究还指出, 功能连接密度 随龄下降最敏感的脑区位于默认网络(后扣带和背侧 后顶叶). 比较不同功能网络衰退程度的研究发现, 老 年人执行控制网络衰退最明显, 其次是背侧注意网 络、默认网络和腹侧注意网络 ${ }^{[75]}$. 在静息态功能网络 内部的连接中, 老年人在额顶控制网络、默认网络和 扣带-盖部网络的功能连接低于青年人 ${ }^{[76]}$. 国内研究发 现, 额叶-脑岛皮层对老年人的默认网络和执行控制网 络起着重要调节作用 ${ }^{[77]}$. 除了正常老化的研究外, 静 息态fMRI研究在MCI及 AD上也有了很多新的发现. 与正常老年人相比， $\mathrm{MCI}$ 老年人呈现出脑局部至长程 的功能连接异常 ${ }^{[78]}$. 在主观认知下降、MCI和 $A D$ 的谱 系中，研究者发现不同的功能网络中心度在不同尺度 上发生了改变 ${ }^{[79]}$.

静息态研究是否能揭示认知老化的神经机制，不 同研究者持有不同观点. 有研究者认为, 在静息状态 下扫描脑，尽管能反映脑自发的功能连接或网络组织 差异, 但由于静息态fMRI研究没有探查扫描时脑的认 知活动, 因此在揭示认知老化的神经机制上, 其作用非 常有限 ${ }^{[0]}$. 其他研究者则认为, 静息态fMRI研究代表 着老化认知神经科学新的研究方向, 在整合研究取向 上发挥着重要作用. 基于任务的脑功能老化研究和基 于自发活动的脑功能老化研究相结合，有助于人们更 好地理解认知老化的神经机制 ${ }^{[81]}$ : (i ) 基于任务的 fMRI研究从功能定位角度出发, 重点关注特定认知任 务引发的局部脑区激活; 静息态fMRI研究可以考察脑 自发活动下不同脑区之间的功能连接，能够从功能整 合的角度考察认知老化的脑机制. (ii) 相对任务fMRI
研究, 静息态fMRI研究可以减少练习效应的影响. (iii) 对于不能完成特定认知任务的老年人, 可以开展静息 态fMRI研究. (iv) 目前国际上有许多共享静息态fMRI 研究数据库, 比如Human Connectome Project ${ }^{[82]}$, Alzheimer's Disease Neuroimaging Initiative (ADNI, http:// adni.loni.usc.edu) 等, 研究者可以获得跨中心和跨站点 的数据, 通过数据整合及分析, 获得更加精确的效果量 数据, 包括组间差异及与认知功能相关的个体差异. 对 于任务态脑成像数据, 获得跨中心的大样本数据相对 较难. (v) 根据去分化理论可知, 老年人完成不同认 知任务时脑激活特异性下降 ${ }^{[63-65]}$; 另外, 不同认知功 能在老化过程中下降速率也不同 ${ }^{[18]}$. 基于认知随龄变 化的复杂性, 采用静息态和任务态fMRI研究相结合的 方法, 能够更好地刻画老化过程中神经认知加工的 机制.

\section{3 脑血流量与脑老化}

认知功能的下降通常伴随着脑血流量的降低, 脑 血流量变化是认知下降病理机制的早期生物学标 记 ${ }^{[83,84]}$. 脑血流量(cerebral blood flow)是指动脉血到脑 组织毛细管床的输送速度 ${ }^{[85]}$. 动脉自旋标记 (arterial spin labeling, ASL)MRI是近来研究者常用的测量脑血 流量的工具, ASL主要通过动脉血水作为内源性示踪 剂进行测量. 研究发现, 老年人整体脑血流量低于青 年人 ${ }^{[86]}$. 然而，不同脑区的脑血流量变化呈现出不同 的模式，其中前额叶、脑岛和尾状核的脑血流量变化 受老化影响最严重 ${ }^{[87]}$.

在静息状态下，研究者发现老年人血氧水平依赖 和脑血流量的动态耦合显著下降，尤其是左侧缘上 回 ${ }^{[88]}$. 研究者也探讨了老年人脑血流量和认知成绩的 关系, 发现海马脑血流量与空间记忆成绩呈正相 关 ${ }^{[89]}$, 左侧题上回脑血流量与整体认知功能呈正相 关 $^{[90]}$. 追踪研究也发现了相似结果. 基线条件下老年 人全脑血流量和全脑灰质体积及执行功能呈正相关; 平均 2.3 年的追踪发现, 脑血流量的降低与脑灰质体积 的下降及加工速度的降低有关 ${ }^{[91]}$. 脑血流量随龄下降 的机制尚不清楚, 可能与神经元数量、神经元大小、 突触密度和/或神经元活性减少有关 ${ }^{[85]}$, 或与动脉二 氧化碳张力的生理变化有关 ${ }^{[92]}$. 未来研究中, 研究者 可进一步关注脑血流量变化在揭示认知老化神经机 制上的作用. 


\section{4 小结}

在老化过程中, 老年人在加工速度、工作记忆、 情节记忆和执行功能等流体智力上均表现出随龄下降 的趋势, 其中情节记忆下降是最为显著的特点. 为了补 偿认知下降，老年人倾向于激活更多的前额叶以完成 认知任务. 在任务难度较低时, 前额叶激活增加可以 补偿老化引起的认知下降; 而在任务难度较高时, 前 额叶激活增加或双侧前额叶激活无法进行成功的补 偿. 研究者从补偿和去分化角度构建了多个认知老化 理论和假说，不同的理论和假说可以解释特定认知老 化领域的研究发现, 并起到互相补充的作用. 基于 MRI、静息态fMRI和ASL MRI的脑老化研究发现了 许多有意义的结果，比如进入老年期后脑灰质体积会 加速萎缩，老年人额顶网络功能连接升高而默认网络 功能连接降低, 脑血流量随龄下降等. 总体来看, 已有 的认知老化理论和假说，较少根据脑结构老化、静息 态研究及脑血流量的发现构建脑老化理论或假说. 未 来的研究可以更多地整合不同层面的研究证据建立认 知老化理论或假说.

\section{2 认知功能干预及脑可塑性}

尽管许多认知功能均随增龄下降，但大量研究表 明, 脑在毕生发展过程中保持着神经可塑性, 部分认 知功能可以保持甚至提升. 已有研究表明，非药物干 预，如运动干预、认知干预以及生活方式干预可以改 善老年人的认知功能.

\section{1 运动干预}

一项纳入了 15 项前瞻性研究的元分析发现，所有 水平的身体活动均能降低老年人的认知下降风险 ${ }^{[93]}$. 该研究清楚表明了运动在预防老年人认知下降中的重 要作用. 最近一项元分析纳入了 23 项考察身体运动与 健康老化的追踪研究, 大多数研究表明身体运动和健 康老化存在正相关，总体效果量达到了显著相关的水 平 ${ }^{[94]}$.

有氧运动训练可以提升老年人海马等脑区的灰质 体积和白质体积 ${ }^{[95 ~ 97]}$. 9年的追踪研究表明, 身体活动 越大的老年人，额叶、枕叶、内嗅皮层和海马的灰质 体积越大 ${ }^{[98]}$, 有氧运动训练后, 有氧适能(aerobic fit- ness)越强的老年人，额叶和题叶白质完整性改变得越 多, 短时记忆的提升也越多 ${ }^{[99]}$. 国内研究者考察了太 极拳训练和八段锦训练的特异性效果，在进行了 12 周 的训练后, 研究者发现与对照组相比, 太极拳组后扣 带与右侧壳核/尾状核的功能连接升高, 而八段锦组内 侧前额叶和眀额/壳核的功能连接降低, 两组表现出了 不同的训练效应 ${ }^{[100]}$. 在为期 1 年的干预研究中, 研究者 把老年人随机分配到有氧运动组(步行组)和非有氧运 动组(灵活性、伸展以及平衡组), 结果发现有氧运动 组老年人高级认知网络的功能效率提升，同时发现执 行控制网络和默认网络的功能连接提升 ${ }^{[101]}$. 相对伸展 运动对照组老年人, 经过半年有氧运动训练的老年人 在背外侧前额叶和顶上回/楔前叶的静息态功能连接 升高 ${ }^{[102]}$. 此外, 国内的研究还发现, 太极拳训练后, 老 年人任务切换扫描时前额叶激活升高 ${ }^{[103]}$, 海马和内侧 前额叶的功能连接提升 ${ }^{[104]}$, 背外侧前额叶的低频振幅 增加 ${ }^{[105]}$.

稳定的脑血流量对脑功能非常重要，然而脑血流 量自成年期起开始逐渐降低 ${ }^{[106]}$, 运动干预可以改变老 年人脑血流变化. 研究者考察了运动训练组和健康教 育对照组老年人在 4 个月训练后的静息态脑血流量变 化, 结果发现, 与对照组相比, 运动训练组老年人海马 血流量显著提升 ${ }^{[107]}$. 12周的有氧训练研究表明, 训练 组老年人显著提升了前扣带回的静息态脑血流量 ${ }^{[108]}$. 老年大师级运动员比久坐老年人在后扣带/楔前叶的 脑血流量高 ${ }^{[109]}$ ，说明运动干预对脑血流量具有长期 影响.

尽管很多研究证明运动干预对老年人认知功能具 有广泛的积极作用，但是有些老年人不能长期坚 持 ${ }^{[110]}$. 运动游戏, 作为一种新型的运动干预方式越来 越受到研究者的关注. 运动游戏具有的运动性、趣味 性和挑战性, 可以极大地增加老年人的兴趣和依从性, 从而提升干预效果 ${ }^{[111]}$. 运动游戏过程中, 玩家需要通 过身体活动与游戏进行交互, 从而达成游戏目标. 研究 表明, 运动游戏能导致能量消耗和心肺活动显著增加, 有助于改善心肺健康; 与跑步机运动和慢跑相比, 运动 游戏的愉悦度更高，是传统有氧运动的可替代方 案 ${ }^{[112]}$. 使用跳舞毯游戏对 65 岁以上的老年人进行 3 个 月的干预后发现，老年人的日常生活能力及对自己平 衡能力的信心显著增加 ${ }^{[13]}$. 把老年人分为平衡运动游 戏组和平衡练习组, 经过6周的干预后发现，两组被试 
在运动灵活性及平衡能力上均有显著提升 ${ }^{[114]}$. 使用任 天堂的Wii Fit游戏对被试进行训练, 发现与对照组相 比, 干预组老年人在身体灵活性和步态、速度上均有 显著改善 ${ }^{[115]}$. 使用运动游戏对老年人进行 12 周(每周 2 次, 每次 $1 \mathrm{~h}$ )运动游戏训练后发现, 与对照组相比, 训练 组的执行控制和加工速度显著改善 ${ }^{[116]}$. 近期的元分析 发现, 运动游戏对临床以及非临床群体的认知提升具 有明显的效果, 对视空间学习和记忆的提升效果最 好 $^{[117]}$. 运动游戏对身体功能及认知功能改善的脑机制 研究还比较少, 未来研究有待加强.

\section{2 认知干预}

认知干预主要指采用非药物干预的方式对认知功 能进行训练, 通常包括认知刺激、认知康复和认知训 练 ${ }^{[118,119]}$. 对于认知健康的老年人来说, 认知训练是常 用的方式 ${ }^{[120]}$. 西雅图老化追踪研究第五周期中, 研究 者开展了目前为止较早也是影响深远的干预研究 ${ }^{[121]}$. 该研究为以后的训练研究特别是ACTIVE(Advanced Cognitive Training for Independent and Vital Elderly)研 究提供了基础. 在ACTIVE研究中, 训练者针对老年人 开展10次训练后, 发现其加工速度、推理和记忆能力 都有提升 ${ }^{[122]}$. 此外, 有研究者提出通过学习复杂的新 技术, 如数码技术和平板电脑可以提升情节记 忆 $^{[123,124]}$. 已有研究发现, 工作记忆训练可以提升老年 人的工作记忆及额顶网络功能 ${ }^{[125,126]}$. 研究者招募了 32 名老年人并随机分配到N-back训练组和空白对照组, 训练组开展了 12 次训练(每次 $45 \mathrm{~min}$ ), 并且让两组被试 都进行了N-back和延迟再认的任务fMRI扫描, 结果发 现对于训练组来说, 在训练后执行N-back任务以及延 迟再认任务时, 右外侧额中回/额上沟的激活均低于对 照组, 这表明训练后老年人用较少的神经资源即可以 获得相同或者更好的行为成绩 ${ }^{[127]}$. 为期 4 个月的空间 导航训练发现, 老年人和青年人在空间导航行为成绩 上均有提升, 但只有青年人在左侧楔前叶和中央旁小 叶的皮层厚度增加, 老年人的皮层厚度并没有增

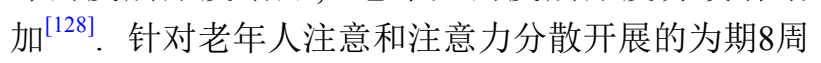
的认知训练研究发现, 训练组老年人前额叶静息态脑 血流比对照组显著提升, 并且血流量的升高与注意力 分散的改善有关 ${ }^{[129]}$. 为期 12 周的推理训练发现, 认知 训练组老年人在内侧眀额和双侧后扣带回的静息态血 流量比对照组显著升高 ${ }^{[130]}$.
近年来, 视频游戏在人们生活中也扮演着越来越 重要的角色. 研究发现, 视频游戏干预能够提升老年 人注意力、记忆力、视空间能力及执行功能等多方面 认知能力 ${ }^{[131 ~ 133]}$. 采用一系列商业认知游戏软件包 (Lumosity)的研究发现, 实验组老年人在所有训练的 认知游戏上均得到了提升, 并且实验组老年人在视空 间工作记忆、拼图任务、情节记忆和短时记忆均有提 升, 而对照组则没有 ${ }^{[134]}$. 经常玩游戏的玩家比不经常 玩游戏的玩家左侧纹状体体积更大 ${ }^{[135]}$. 毕生玩视频游 戏的时间越长, 双侧海马旁回特别是内嗅皮层以及枕 叶/顶下小叶的体积越大 ${ }^{[136]}$. 老年视频游戏玩家的执 行功能成绩显著高于非视频游戏玩家, 且在完成Flanker任务时额顶区域激活升高 ${ }^{[137]}$. 具有视频游戏经验 的老年人, 在左侧顶下回、小脑及舌回的低频振幅高 于对照组老年人 ${ }^{[138]}$.

\section{3 生活方式影响及干预}

近来，研究者越来越关注生活方式对认知老化的 影响. 研究者发现, 认知参与的休闲活动, 如阅读、小 组讨论、计算机使用、玩纸牌游戏、填字谜、玩乐器 以及学习第二外语等均能有效地延缓认知下降 ${ }^{[139 \sim 143]}$. 对330名50 89岁中老年人的研究表明, 忙碌的生活方 式与更好的加工速度、工作记忆、情节记忆、推理和 晶体知识等认知能力有关 ${ }^{[144]}$. 一项纳入了 1211 名中老 年人的研究发现, 健康饮食、更多的身体活动、丰富 的智力以及更多的社会活动与较低的 $\mathrm{MCI}$ 发病风险相 关 $^{[145]}$. 另一项采用 259 名横向数据的成年毕生发展 (18 81岁)研究表明, 休闲娱乐活动有助于成功老 化 ${ }^{[146]}$. 一项长达 80 年的追踪研究发现, 在控制人口学 变量及儿童期认知能力后, 中年期的休闲活动和认知 能力呈正相关，晚年期身体活动水平越高、认知下降 越少 ${ }^{[147]}$. 探讨生活方式因素和认知功能的研究发现, 有氧代谢能力越强和日常身体活动越多的老年人, 执 行功能、加工速度和言语记忆成绩越好; 越符合高血 压防治计划(Dietary Approaches to Stop Hypertension, DASH)饮食的老年人, 言语记忆成绩越好 ${ }^{[148]}$.

近来, 研究者也开展了生活方式干预对认知老化 影响的研究. 研究者把 160 名久坐不爱运动的老年人 随机分配到了有氧运动组、DASH饮食组、有氧运动 和DASH联合训练组以及健康教育对照组, 结果发现, 有氧运动和DASH联合训练组在执行功能整合分数上 
提升最高. 此外, 心血管风险越低、钠摄入越少的老年 人, 执行功能提升越多 ${ }^{[149]}$. 研究者分别对 631 名老年人 开展了为期两年的多领域干预，包括饮食建议、力量 训练、认知训练及心血管风险因素管理, 对照组包含 629 名被试，所有被试的年龄介于60 77岁. 当研究者 对饮食干预进行分析时，发现生活方式干预组老年人 在第一年追踪和第二年追踪时维生素和矿物质摄取上 均显著高于对照组, 而这有助于防止认知下降 ${ }^{[150]}$. 国 内对 555 名MCI老年人的研究表明, 结构化生活方式干 预有助于老年人延迟回忆和词语流畅性成绩的提升, 依从性越好的老年人认知成绩提升得越多 ${ }^{[151]}$. 基于 460名60 95岁老年人 6 个月的生活方式干预的研究表 明, 与空白对照组相比, 生活方式干预组在躯体疼痛、 活力、社会功能、心理健康、生活满意度以及抑郁症 状方面均有显著改善 ${ }^{[152]}$.

饮食也会影响脑结构和功能老化. 元分析研究表 明, 维生素D缺失与脑体积降低有关 ${ }^{[153]}$. 维生素B12、 维生素D、锌、单不饱和及多元不饱和脂肪摄入量与 脑体积呈显著正相关, 而饱和脂肪、反式饱和脂肪、 胆固醇和钠摄入量与脑体积呈显著负相关 ${ }^{[154]}$. 基于 116 名老年人的研究表明, $\omega-3$ 和 $\omega-6$ 多元不饱和脂肪酸 以及胡萝卜素与静息态功能网络效率呈显著正相关. $\omega-3$ 多元不饱和脂肪酸调节额顶网络和一般智力, $\omega-6$ 多元不饱和脂肪酸和番茄红素调节背侧注意网络和执 行功能 ${ }^{[155]}$. 研究者通过正电子发射断层成像 (positron emission computed tomography, PET)考察了49名 25 72岁成年人膳食营养素和脑生物学标记的关系, 结果发现, $\beta$-胡萝卜素和叶酸与顶下小叶、外侧题 叶、内侧前额叶以及后扣带的葡萄糖代谢呈显著正相 关 ${ }^{[156]}$. 追踪研究表明, 对地中海饮食模式依从性较低 的中年人，其后扣带和额叶皮层的葡萄糖代谢率下降 更快 ${ }^{[157]}$.

目前，生活方式干预对老年人脑可塑性的研究还 较少，但该领域已经引起了研究者越来越多的重视. 2019年7月, 加州大学伯克利分校从美国老龄化研究 所获得了一项为期 5 年、总额达 4700 万美元的项目资 助. 该项目计划将脑成像技术整合到“通过生活方式干 预降低风险来保护脑健康的美国阿尔茨海默病联合会 研究”(The Alzheimer's Association U.S. Study to Protect Brain Health Through Lifestyle Intervention to Reduce Risk, U.S.POINTER, https://news.berkeley.edu/ 2019/07/29/47-million-grant-to-explore-how-a-healthylifestyle-changes-the-aging-brain/), 通过多中心临床试 验考察调节多个风险因素的生活方式干预(如健康饮 食、身体活动、健康指导、社会化和智力挑战等)是 否可以改善老年人思维和记忆等认知衰退和痴呆症 状. 该项目计划利用先进的神经影像技术探索生活方 式干预对被试的全局和局部脑形态和血流的影响，同 时还会监测影响脑血管疾病的各项指标，并将采用 PET追踪脑内 $\beta$ 淀粉样蛋白和 $\operatorname{tau}$ 蛋白水平. 可以预见, 该项目的实施将会提供丰富的生活方式干预对老年人 脑可塑性影响的证据.

\section{4 小结}

已有研究表明, 运动干预、认知干预和生活方式 干预均有助于保持或改善老年人的认知与脑老化. 大 量研究认为, 运动干预可以改善脑结构和功能老化, 也有较多研究认为认知干预能起到改善效果. 以计算 机、平板电脑、手机或其他平台为媒介的数字化干 预, 是目前流行的干预方式, 有望为老年人脑结构和功 能老化的改善带来新的希望. 近年来, 研究者开始重视 生活方式对健康老化的影响, 并发现一些生活方式因 素和生活方式干预有助于延缓老年人的认知下降. 根 据已有的研究发现，对老年人来说，坚持规律的运 动、多做一些认知参与的活动、保持健康的生活方式 及饮食模式, 可以帮助其保持认知功能.

另外, 在已有研究中, 不同研究会得出不同甚至是 相反的结果, 这可能是由于取样方法、样本量大小、 个体差异因素(比如年龄、性别、受教育程度、基线 认知能力、动机、是否相信训练有效果等)等造成的. 未来干预研究，除报告脑可塑性(比如脑区激活、功能 连接、灰质体积、皮层厚度等)结果之外，同时报告脑 可塑性和认知能力提升的关系, 将有助于读者更好地 理解研究发现. 此外, 大样本、多中心的追踪研究以 及系统的元分析研究也有助于获得较为客观的研究 结论.

\section{3 老化认知神经科学未来发展方向预测和 展望}

脑成像研究在老化认知神经科学中扮演着越来越 重要的角色. 无论是认知老化理论的进展、脑可塑性 
的训练研究还是成人毕生发展的认知神经科学研究, 基于脑成像研究的证据都起着重要的作用. 关于老化 认知神经科学未来的发展, 本文做出了如下预测和展 望(图1).

\section{1 认知老化理论的发展}

截至目前，老年人脑结构和功能随龄变化与认知 下降的关系并不清楚, 揭示认知老化与脑功能和结构 老化的关系是老化认知神经科学研究的重要目标 ${ }^{[158]}$. 探讨脑功能与认知老化的系统综述发现，70\%的研究 报告了老年人脑激活程度(特别是前额叶)与认知能力 存在正相关 ${ }^{[159]}$. 老年人默认网络前部脑区活动与执行 功能具有显著负相关, 而默认网络后部脑区与数字表 征显著正相关 ${ }^{[160]}$. 默认网络功能连接的随龄下降与工 作记忆成绩呈显著正相关 ${ }^{[161]}$. 基于73名36 86岁健康 成年人的功能连接和神经心理测试发现, 腹侧注意网 络的左侧脑岛和背侧前扣带的功能连接与执行功能和 额叶评定量表显著相关 ${ }^{[162]}$. 未来尚需要更多大样本纵 向研究考察脑结构和功能老化与特定认知能力随龄变 化的关系.

随着研究的深入以及多学科的交叉融合, 研究者 对于认知老化的认识也在加深, 因此认知老化的理论 也在动态发展着. 在认知心理学作为研究主流的时候, 出现了一批基于行为学研究的认知老化理论或模型, 如认知老化的加工速度理论 ${ }^{[29,30]}$ 、抑制能力减弱假 说 ${ }^{[37,38]}$ 、前额叶功能老化理论 ${ }^{[40,41]}$ 、环境支持假 说 ${ }^{[163,164]}$ 以及选择、优化、补偿模型 ${ }^{[165]}$. 随着认知神 经科学的兴起及越来越多神经影像学证据的出现, 研 究者提出了一批基于神经影像学的认知老化理论或模 型, 如半球非对称性下降假说 ${ }^{[5]}$ 、老化的前后转移模 型 ${ }^{[5]}$ 、神经环路的补偿相关利用假说 ${ }^{[60]}$ 、去分化假 说 ${ }^{[63 \sim 65]}$ 及老化与认知的脚手架理论 ${ }^{[61,62]}$ 等. 近年来, 关 于老化脑功能网络的研究对现有认知老化模型提出了 极大的挑战, 亟需深入开展认知老化的功能网络研究, 以提升对认知老化神经机制的认识. 同时, 在构建认知 老化理论或模型时，也要结合脑结构老化、静息态 fMRI及脑血流量的研究发现, 完善现有的或建立新的 认知老化理论或模型.

\section{2 从成人毕生发展视角探讨脑老化}

已有的关于认知老化的脑成像研究多建立在青年
人和老年人对照研究基础上. 采用老年组-青年组对照 的研究, 样本量通常较小且不具有代表性, 会限制研究 结论的推广及对认知老化的理解. 近年来, 越多越多的 研究开始从成人毕生发展视角研究认知与脑老化. 利 用情节记忆编码任务考察成人毕生发展脑活动的研究 发现, 记忆成绩差的个体在中年时就表现出了负相继 记忆效应(negative subsequent memory), 而记忆成绩 好的个体则直到老年阶段才表现出这一特点 ${ }^{[166]}$. 不同 年龄段被试在执行语义任务激活的脑活动中出现了较 大的差异, 中年人(40 59岁)和老年人(60 79岁)的差异 最大, 而青年人 $(20 \sim 39$ 岁 $)$ 和中年人, 以及老年人和老 老年人 $(80 \sim 89$ 岁 $)$ 之间的差异较小 ${ }^{[167]}$. 与久坐的人相 比, 中年时身体活动更多的人在老年期的灰质体积更 $大^{[168]}$.

个体健康细微或深远的变化往往发生在中年, 包 括心血管生理和荷尔蒙变化的衰减, 以及影响健康的 生活方式的变化 ${ }^{[82]}$. 在研究认知老化脑机制时, 纳入 青年人、中年人和老年人，系统考察成人毕生发展过 程中认知老化的神经基础, 能够更加准确地了解脑结 构和脑功能老化的发生时间; 可以揭示从青年到老年 的过程中, 脑结构和脑功能的老化是逐渐发生的, 还 是随着年龄的增长呈现出非线性的变化 ${ }^{[169]}$. 目前, 已 有少数研究者开始探索人脑功能连接的毕生发展轨 线. 采用基于体素的功能同伦方法刻画了214名7 85岁 的被试半球功能连接, 结果发现, 毕生发展轨线在全局 水平上呈现出U型, 而在局部脑区上则表现出了明显 的区域差异 ${ }^{[170]}$. 成人毕生发展(20 89岁)的静息态脑 功能网络变化表明, 在脑网络内部连接与网络之间的 连接上, 青年人相对平衡, 随着年龄的增长, 脑功能的 系统分化能力开始下降 ${ }^{[171]}$. 采用毕生发展(7 85岁)静 息态脑成像数据考察功能网络的发展轨线发现, 随着 年龄增长, 网络内部的功能连接下降, 而网络之间的 功能连接上升 ${ }^{[172]}$. 未来研究中, 亟待开展大样本、长 期追踪的研究, 从成人早期开始进行认知、脑结构、 脑功能等层面的长期追踪, 更深入地探讨认知与脑老 化的机制.

从成人毕生发展视角开展认知老化研究, 创建成 人毕生发展的脑老化曲线, 有助于揭示成人不同阶段 表现出相似症状(比如成年抑有症和老年抑郁症等)的 病理生理共同点和差异性, 从而有利于对神经或精神 疾病开展早期诊断和早期干预 ${ }^{[173]}$. 绘制成人毕生发展 

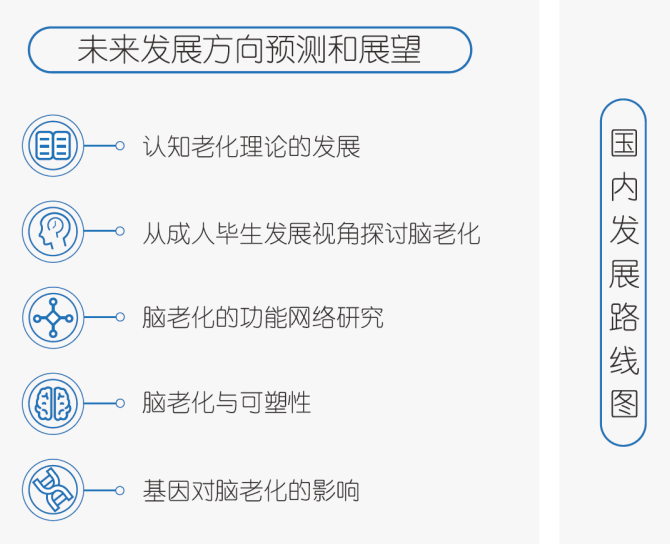

开展大样本，长期追踪的成人 毕生发展研究, 建立成人毕生 发展行为-基因-脑数据库
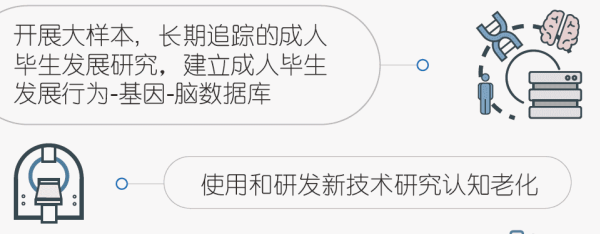

深入探索有效的脑功能改善方法
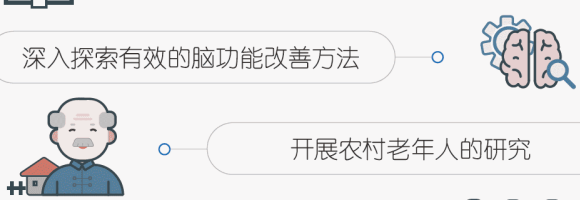

$\circ$

开展农村老年人的研究

开展临床老年群体的研究

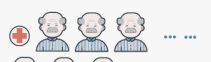
888

图 1 老化认知神经科学未来发展方向预测和展望及国内发展路线图(网络版彩图)

Figure 1 Forecast and prospect of the future development of aging cognitive neuroscience and the domestic development roadmap (color online)

的脑结构和功能常模曲线, 可以评估疑似神经疾病或 精神疾病个体的脑结构和功能特点 ${ }^{[174]}$. 例如, 已有研 究发现, $\mathrm{AD}$ 病人经历着缓慢而又渐进性的脑萎缩. 然 而, 健康老年人的脑老化曲线和 $\mathrm{AD}$ 病人的脑老化曲线 在何时呈现不同，以及在脑老化的哪个时间节点寻找 $\mathrm{AD}$ 的脑成像生物学标记, 皆有赖于通过成人毕生发展 研究获取答案 ${ }^{[175]}$. 此外, 由于一些重要的认知功能从 中年期甚至青年期就开始下降, 脑成熟和老化曲线有 助于确定干预的最佳时期 ${ }^{[176]}$, 有助于促进个体的健康 老化甚至成功老化.

\section{3 脑老化的功能网络研究}

目前对认知老化神经机制的理解, 多建立在认知 任务诱发的脑区活动强度的组间比较上, 较少从脑功 能网络的层面去理解认知老化的机制.

人脑功能连接组学正在以一种全新的角度从系统 水平上认识和理解人脑及其发展变化 ${ }^{[177]}$. 系统神经科 学认为, 脑由不同的功能网络组成, 是一个具有高度灵 活性和适应性的系统. 随着认知加工的进程, 该系统不 断进行动态调整 ${ }^{[178]}$. 系统神经科学不仅有助于描述正 常及异常的脑网络 ${ }^{[179]}$, 而且可以开发预测神经退行性 疾病及其进展的神经影像学标记, 有助于揭示认知衰 老的神经机制. 当前, 人脑功能网络研究已经对“人脑 作为一个复杂系统如何工作的宏观机制”有了初步理 解 ${ }^{[180]}$. 额顶网络、背侧注意网络和腹侧注意网络三大 网络被认为是人脑的控制系统, 负责外界环境(通过视 觉、体感运动和边缘网络)和内部环境(通过默认网络)
的自适应信息交互, 是认知加工的核心枢纽系统 ${ }^{[181]}$. 对认知老化任务fMRI研究的系统综述发现, 在老年人 激活升高的功能网络中, 控制系统占比超过 $70 \%$. 在完 成认知任务时, 老年人激活了较多的控制系统, 说明控 制系统的调控功能在认知老化过程中扮演着重要角

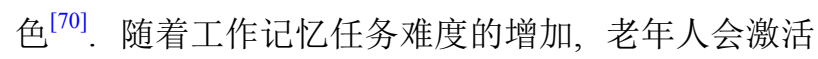
更多的皮层功能网络, 且工作记忆能力越强的老年人 表现出更好的功能网络整合, 这可能是老化功能网络 重组的机制 ${ }^{[182]}$. 通过比较老年人和青年人在伦敦塔任 务中的额顶网络和默认网络活动特点, 研究者发现, 在 任务难度较小时, 老年人和青年人均同时激活了额顶 控制网络并且抑制了默认网络; 随着难度增加, 老年 人的额顶网络活动下降, 同时对默认网络的抑制能力 也下降. 据此, 研究者提出了认知老化的默认网络-执 行控制网络耦合假说 ${ }^{[68]}$. 从脑功能网络角度研究认知 老化, 正在开启认知老化研究的新篇章, 其研究发现 已经突破了现有的以脑激活为主要依据的认知老化模 型 ${ }^{[183]}$. 随着对认知老化脑功能网络机制认识的深入, 研究者将构建更多的基于功能网络的认知老化模型, 或者对现有认知老化模型进行修订, 以纳入认知老化 功能网络的研究发现.

\section{4 脑老化与可塑性}

在未来研究中, 研究者需要投入更多的时间, 从成 人毕生发展角度探讨提升认知的方法. 目前出现的大 量干预技术, 比如运动干预、认知训练、游戏训练、 生活方式干预等均是以提升认知功能或延缓认知下降 
特别是记忆下降为目的. 然而, 认知训练、游戏训练及 生活方式干预等仍然是非常年轻的领域，尚需要更多 严格的科学研究来探讨训练效果的稳定性、迁移性以 及持续性. 认知老化领域的研究者需继续保持研究兴 趣, 继续开展更多的训练研究进行深入探讨.

对脑老化可塑性机制的探索将有助于提升训练效 果的稳定性、迁移性和持续性. 前人研究表明, 多巴胺 水平可能是影响神经可塑性的重要原因. 老年人多巴 胺水平的改变与工作记忆和抑制控制下降有关 ${ }^{[184,185]}$. 尽管已有研究表明, 认知训练可以增加纹状体的多巴 胺 ${ }^{[186,187]}$, 老年人升高的多巴胺整合能力对认知功能 保持起补偿作用 ${ }^{[188]}$. 但是，研究者认为，老年人较低 的多巴胺水平限制了其认知资源的上限及神经可塑性 的程度 ${ }^{[189]}$. 尽管认知训练可以改变部分脑区的多巴胺 水平，但是多巴胺水平变化与脑结构和脑功能可塑性 变化之间的关系, 尚需要更多研究来解答.

个体差异也是可塑性研究非常重要的问题. 已有 研究发现，年龄、受教育水平和基线成绩可以预测训 练效应 ${ }^{[190]}$. 国内研究者发现，流体智力基线水平高的 被试, 工作记忆训练改善效果更好 ${ }^{[191]}$. 认知训练中获 益最多的老年人, 在训练前表现出了更多脑网络的分 离(即模块化), 特别是与训练的认知功能密切相关的 网络 ${ }^{[192]}$. 脑模块性能够以灵活高效的方式组织和传达 信息 ${ }^{[193]}$, 具有高脑模块化的老年人会表现出更大的训 练效应和更广泛的迁移效应. 未来研究可以深入探讨 脑结构、脑血流量、脑功能激活及脑功能网络对运动 干预、认知干预及生活方式干预效果的预测效应.

\section{5 基因对脑老化的影响}

越来越多的研究表明，基因对认知和脑功能的影 响随年龄增长而变大. 一项基于 27 项研究的元分析表 明，遗传会不同程度地加速老年人空间加工、知觉速 度、工作记忆等认知能力的下降 ${ }^{[194]}$. 到了老年之后, 携带不良基因型的老年人会呈现出加速的认知下降和 脑老化 ${ }^{[195]}$. APOE, BDNF, COMT以及 KIBRA基因均影 响认知 ${ }^{[196]}$. 有证据表明, 不良基因对老年人会产生更 大的影响 ${ }^{[197]}$. 国内研究表明, 携带KIBRA C等位基因 的老年人，其前额叶及海马旁回的灰质体积低于携带 KIBRA TT等位基因的老年人 ${ }^{[198]}$. 与非携带者相比, 老年 $A P O E \varepsilon 4$ 携带者脑白质结构网络整体连接效率与 静息态功能网络的连接效率下降 ${ }^{[199]}$; 老年 $A P O E \varepsilon 4$ 携
带者认知功能受损时，整体白质网络连接及网络内右 侧海马的节点效率显著下降 ${ }^{[200]}$. 在进行图片编码加工 时，携带 $A P O E \quad \varepsilon 4$ 基因的老年人比对照组老年人激活 了更多的双侧梭状回、右侧顶叶、右内侧前额叶以及 左侧额中回 ${ }^{[201]}$. 未来尚需开展更多的研究深入探索老 化易感基因对特定认知功能、脑结构和脑功能的影 响, 以及是否可以预测老年人的认知下降.

\section{4 国内发展路线图}

目前, 在认知老化理论构建上, 主流的老化理论均 是西方研究者基于西方老年人的研究提出的. 自 1977 年起，每隔5 8年(1996年起每隔5年)美国会发布新版 《老化生物学手册》(Handbook of Biology of Aging)、 《老化心理学手册》(Handbook of Psychology of Aging)和《老化与社会科学手册》(Handbook of Aging and Social Sciences), 届时根据老化生物学、 老年心理学以及老化与社会科学的最新进展, 邀请各 个分支领域的专家撰写相应主题，及时总结已有进展 并对未来研究做出展望. 为考察中国老年心理学学科 发展的全貌, 中国科学院心理研究所韩布新团队 ${ }^{[202]}$ 对 中国科学引文数据库发表的 885 篇老年心理学论文进 行了计量学分析, 发现采用实验研究的文献比例偏低, 论文发表的地区分布不均衡，与当前社会日益增长的 需求之间尚有差距.

根据国内研究现状, 在如下方面开展老化认知神 经科学研究, 将有助于实现国内老化认知神经科学的 发展，提升老年生活质量，服务国家养老保障体系建 设(图1).

(1) 开展大样本、长期追踪的成人毕生发展研究, 建立中国成人毕生发展行为-基因-脑数据库. 美国、 德国、瑞典、澳大利亚等发达国家均开展过大样本、 长期追踪的老化研究, 尽管这些长期追踪研究多是从 行为层面开展的. 相比之下, 国内认知老化研究的力 量还较为薄弱, 目前大规模的长期追踪研究还较少. 因此, 建立国内成人毕生发展脑与认知数据库意义重 大. 绘制脑功能/结构成人毕生发展曲线, 有助于揭示 脑随年龄增长的发展及动态变化规律, 为脑老化程度 的评估提供客观的测量指标. 同时，可以监测脑老化 发展态势, 为脑异常老化的早期识别及脑退行性病变 的诊断提供帮助. 此外, 脑功能/结构老化曲线有助于 
检验和评估脑功能的改善效果，为干预措施的改进及 选择提供有效参考. 中国健康与养老追踪调查(China Health and Retirement Longitudinal Study, CHARLS) 在2011年开展了全国基线调查，并在随后开展了多次 追踪调查，迄今收集 1.9 万 45 岁及以上中老年人家庭和 个人的微观数据, 主要包括个人基本信息, 家庭结构和 经济支持，健康状况，体格测量，医疗服务利用和医疗 保险，工作、退休和养老金、收入、消费、资产，以及 社区基本情况等情况(http://charls.pku.edu.cn/pages/ about/111/zh-cn.html). 该调查为推动我国老龄化问题 的跨学科研究提供了重要的基础和支撑. 北京师范大 学张占军团队 ${ }^{[203]}$ 在2008年开展了“北京BABRI老年脑 健康促进计划”(Beijing Aging Brain Rejuvenation Initiative, BABRI)社区临床队列研究, 该研究计划通过 20 年完成 10000 名社区中老年样本的数据采集，其中 计划采集 5000 多例多模态磁共振成像数据. 该研究目 前已完成了 7000 多人次的社区中老年人群数据采集, 为建立中国老年认知与脑老化基础数据库、探索中国 老年人群的认知与脑老化规律贡献了重要力量.

(2) 使用和研发新技术研究认知老化. MRI技术的 发展极大地促进了老化认知神经科学的进展. 未来, 具 有更高时空分辨率的神经成像技术将会进一步提升人 们对脑老化的认识. 能够实时监控神经递质活动和基 因表达的技术，对于理解认知老化的神经机制也具有 重要意义. 此外, 开发能够记录人们在实际场景工作 时神经活动的工具, 将有助于从实验室研究转移到真 实生活中进行研究, 将增强研究结果的生态效度和推 广性 ${ }^{[204]}$.

(3) 深入探索有效的脑功能改善方法. 近来, 有研 究认为神经刺激可以改善认知功能. 在国内老年人口 日益庞大的现实背景下，在国际技术封锁和技术壁垒 的背景下，能否率先开发出新的干预技术是关乎老年 人生活质量、医疗卫生负担的大事. 有研究者探索了 经硕直流磁刺激(transcranial direct current stimulation, tDCS) 一一 一种非侵入式的脑刺激技术, 对老年人认知 功能的改善作用，发现tDCS 可以改善青年人以及老年 人的记忆功能 ${ }^{[205]}$. 如果这些研究发现被认为是可靠并 且可以重复，那么脑刺激的方法在认知老化干预中将 起重要作用. 未来的研究需要继续验证 $\mathrm{tDCS}$ 是否可以 有效改善老年人的认知能力. 此外, tDCS和认知干预 相结合, 是否能产生比单独 $\mathrm{tDCS}$ 或认知干预更好的训
练效果, 也值得探索.

如前所述，大量研究发现运动干预和认知干预可 以延缓老年人的认知下降. 运动干预和认知干预结合, 是否能产生更好的训练效果、更长的保持时间以及更 广泛的迁移效应, 也是非常值得研究的问题. 未来, 设 计更加严谨、样本量更大且考察训练剂量效应、迁移 效应和保持效应的研究有望给出较为明确的答案.

经过严格科学验证的闭环视频游戏在认知老化 干预中也具有重要前景 ${ }^{[206]}$. 闭环视频游戏是指融合 快速、实时、行为驱动、适应性挑战及行为反馈于 一体的交互式视频游戏，经过系统开发及验证的闭循 环视频游戏能够惠及日益增长的老年人群. 闭环视频 游戏训练和运动干预相结合, 也是未来重要的研究 方向.

(4) 开展农村老年人的研究. 根据我国2015年第四 次中国城乡老年人生活状况抽样调查可知, 城镇老年 人的人均年收入是农村老年人的 2.1 倍，而农村老年人 的受教育水平也低于城镇老年人 ${ }^{[207]}$. 前人研究表明, 丰富和复杂的环境可以改善认知 ${ }^{[208 ~ 210]}$. 农村老年人 经济和文化的相对贫困可能会影响其生活环境的丰富 性, 并对认知老化产生不利影响. 国内研究表明, 农村 地区痴呆发病率 $(6.05 \%)$ 要显著高于城市 $(4.40 \%)^{[211]}$. 国内研究者比较了 555 名城市和农村老年人在简易精 神状态检查表(mini-mental state exam, MMSE)的得分, 发现城市老年人在MMSE总分、定向力、注意力和计 算力、记忆力、回忆能力和语言能力上均优于农村老 年人 ${ }^{[212]}$. 其他研究者也发现城市老年人在蒙特利尔认 知评估量表的得分显著高于农村老年人 ${ }^{[213]}$.

老化认知神经科学研究经常使用事件相关电位及 脑成像设备, 这些设备多安放在大学和研究机构, 由于 便携性较低或不易移动等原因，已有的老化认知神经 科学研究多以城市老年人为样本开展. 由于农村老年 人和城市老年人认知储备不同，在城市老年人上发现 的认知及脑老化机制及有效的干预手段，对农村老年 人是否也同样适用并有效，还需要进一步验证. 此外, 许多中国老年学和老年医学学会评选认定的长寿之乡 都在农村. 对长寿之乡老年人开展大样本追踪研究有 利于深入探讨成功老化的认知与脑机制.

(5) 开展临床老年群体的研究. 随着年龄的增长, 老年人罹患高血压、糖尿病、抑郁症、 $\mathrm{AD}$ 和各种类 型痴呆的风险也在增大. 根据国内开展的高血压患病 
率的流行病学调查, 65 74岁组和75岁以上组的患病率 分别为 $55.7 \%$ 和 $60.2 \%{ }^{[214]}, 60$ 岁以上老年人糖尿病的 患病率为 $20.2 \%{ }^{[215]}$. 近期的元分析研究表明, 国内老 年抑郁症的患病率为 $2.7 \%{ }^{[216]}$. 国内 65 岁以上MCI、痴 呆和 $\mathrm{AD}$ 患病率分别为 $20.8 \% 、 5.14 \%$ 和 $3.21 \%{ }^{[217]}$. 已 有研究表明, 高血压和糖尿病均可以预测老年人的认 知下降 ${ }^{[218,219]}$ ，老年抑郁症患者在情节记忆、执行功 能及加工速度方面也均出现了显著下降 ${ }^{[220]}$, 认知下降 也是 MCI、 $\mathrm{AD}$ 和其他类型痴呆的主要诊断标准和重 要的临床表现. 在开展临床老年群体神经科学研究时, 由于临床群体具有特殊性，要重视基因、环境和伴发 疾病对认知老化的影响. 以高血压病为例, 在东亚人
群上，高血压全基因组关联分析报道了与舒张压或收 缩压相关的基因位点, 包括 $S T 7 L-C A P Z A 1, F I G N$ $G R B 14, E N P E P$ 和 $T B X 3^{[221]}$; 我国居民日常钠盐摄入量 显著高于欧美国家居民, 北方地区高于南方地区, 而钠 盐摄入过多和/或钾摄入偏低, 是我国居民高血压发病 的重要危险因素 ${ }^{[222]}$; 老年高血压患者通常也会有一些 伴发疾病, 如冠心病、脑血管疾病、肾功能不全及糖 尿病等 ${ }^{[223]}$. 因此, 开展临床老年群体认知神经科学研 究需综合考虑上述因素对认知与脑老化的影响. 积极 开展临床老年群体的认知神经科学研究, 探索临床老 年群体认知下降的改善方法, 不仅具有非常重要的现 实意义, 也助于健康中国计划的实施.

致谢＼cjkstart在前期访谈中, 中国科学院心理研究所韩布新研究员、北京师范大学王大华教授和彭华茂教授、江苏师范大学 朱祖德教授、陕西师范大学张宝山教授和李西营副教授在老化认知神经科学未来发展方向及国内发展路线图上提供 了宝贵的信息和建议, 在此表示真诚的感谢. 感谢审稿专家提出的修改建议, 对本文完善起了非常重要的作用.

\section{参考文献}

1 United Nations, Department of Economic and Social Affairs, Population Division. World Population Ageing 2019: Highlights (ST/ESA/SER.A/ 430). 2019

2 National Bureau of Statistics of the People's Republic of China. The 2019 Statistical Bulletin of the People's Republic of China on National Economic and Social Development (in Chinese). 2020 [中国人民共和国国家统计局. 中华人民共和国2019年国民经济和社会发展统计公 报. 2020]

3 Bishop N A, Lu T, Yankner B A. Neural mechanisms of ageing and cognitive decline. Nature, 2010, 464: 529-535

4 Hedden T, Gabrieli J D E. Insights into the ageing mind: a view from cognitive neuroscience. Nat Rev Neurosci, 2004, 5: 87-96

5 Raz N, Rodrigue K M. Differential aging of the brain: Patterns, cognitive correlates and modifiers. Neurosci Biobehav Rev, 2006, 30: 730-748

6 Gong X, Xiao H, Wang D. Emotional valence of stimuli modulates false recognition: Using a modified version of the simplified conjoint recognition paradigm. Cognition, 2016, 156: 95-105

7 Peng H, Gao Y, Mao X. The roles of sensory function and cognitive load in age differences in inhibition: evidence from the stroop task. Psychol Aging, 2017, 32: 42-50

8 Liu H H. Age-related effects of stimulus type and congruency on inattentional blindness. Front Psychol, 2018, 9: 794

9 Liu H H, An Y Y, Li H M, et al. Development and aging of decision-making rationality under risk framework (in Chinese). Chin J Contemp Neurol Neurosurg, 2014, 14: 186-191 [刘涵慧, 安艳艳, 李慧敏, 等. 风险框架情境下决策理性的老化研究. 中国现代神经疾病杂志, 2014, 14: 186-191]

10 Zhang J X, Hai L G, Li H J. Quantification of spatial navigation and application research in cognitive aging (in Chinese). Adv Psychol Sci, 2019, 27: 2019-2033 [张家金金, 海拉干, 李会杰. 空间导航的测量及其在认知老化中的应用. 心理科学进展, 2019, 27: 2019-2033]

11 Owsley C, Sloane M, McGwin Jr. G, et al. Timed instrumental activities of daily living tasks: relationship to cognitive function and everyday performance assessments in older adults. Gerontology, 2002, 48: 254-265

12 Harada C N, Natelson Love M C, Triebel K L. Normal cognitive aging. Clin Geriatr Med, 2013, 29: 737-752 
15 Fillit H M, Butler R N, O’Connell A W, et al. Achieving and maintaining cognitive vitality with aging. Mayo Clin Proc, 2002, 77: 681-696

16 Park D C, Lautenschlager G, Hedden T, et al. Models of visuospatial and verbal memory across the adult life span. Psychol Aging, 2002, 17: 299-320

17 Salthouse T A. Selective review of cognitive aging. J Int Neuropsychol Soc, 2010, 16: 754-760

18 Hartshorne J K, Germine L T. When does cognitive functioning peak? The asynchronous rise and fall of different cognitive abilities across the life span. Psychol Sci, 2015, 26: 433-443

19 Li D M, Chen T Y, Li G Y, et al. Life cognitive development: its separability and individual difference (in Chinese). Psychol Sci, 2004, 27: 1288-1290 [李德明, 陈天勇, 李贵芸, 等. 认知能力的毕生发展及其分离性和个体差异性研究. 心理学报, 2004, 27: 1288-1290]

20 Ma Y X, Zhu Y, Chen Y M, et al. Study on physiological and overall aging dynamic changes in memory and cognitive function in the elderly and elderly (in Chinese). Chin J Gerontol, 2005, 11: 1303-1306 [马永兴, 竺越, 陈韵美, 等. 老年及高龄老人记忆及认知功能生理增龄性及 整体增龄性动态改变的研究. 中国老年学杂志, 2005, 11: 1303-1306]

21 Singer T, Verhaeghen P, Ghisletta P, et a. 1 The fate of cognition in very old age: six-year longitudinal findings in the Berlin Aging Study (BASE). Psychol Aging, 2003, 18: 318-331

22 Anstey K J, Luszcz M A, Sanchez L. Two-year decline in vision but not hearing is associated with memory decline in very old adults in a population-based sample. Gerontology, 2001, 47: 289-293

23 Karlamangla A S, Miller-Martinez D, Aneshensel C S, et al. Trajectories of cognitive function in late life in the United States: demographic and socioeconomic predictors. Am J Epidemiol, 2009, 170: 331-342

24 Craik F I M. Age differences in human memory. In: Birren J E, Schaie K W, eds. Handbook of The Psychology of Aging. New York: Van Nostrand Reinhold, 1977. 384-420

25 Moscovitch M, Winocur G. The neuropsychology of memory and aging. In: Craik F I M, Salthouse T A, eds. Handbook of Aging: Cognition. Hillsdale: Erlbaum, 1992. 315-372

26 Craik F I M, Jennings J M. Human memory. In: Craik F I M, Salthouse T A, eds. Handbook of Aging and Cognition. Hillsdale: Erlbaum, 1992. 51-110

27 Parkin A J, Walter B M, Hunkin N M. Relationships between normal aging, frontal lobe function, and memory for temporal and spatial information. Neuropsychology, 1995, 9: 304-312

28 Spencer W D, Raz N. Differential effects of aging on memory for content and context: A meta-analysis. Psychol Aging, 1995, 10: 527-539

29 Salthouse T A. Speed of behavior and its implications for cognition. In: Birren J E, Schaie K W, eds. Handbook of The Psychology of Aging. New York: Van Nostrand Reinhold Company, 1985. 400-426

30 Salthouse T A. The processing-speed theory of adult age differences in cognition. Psychol Rev, 1996, 103: 403-428

31 Rabbitt P, Scott M, Lunn M, et al. White matter lesions account for all age-related declines in speed but not in intelligence. Neuropsychology, 2007, 21: 363-370

32 Turken A, Whitfield-Gabrieli S, Bammer R, et al. Cognitive processing speed and the structure of white matter pathways: convergent evidence from normal variation and lesion studies. NeuroImage, 2008, 42: 1032-1044

33 Rabbitt P, Mogapi O, Scott M, et al. Effects of global atrophy, white matter lesions, and cerebral blood flow on age-related changes in speed, memory, intelligence, vocabulary, and frontal function. Neuropsychology, 2007, 21: 684-695

34 Li S C, Lindenberger U, Sikström S. Aging cognition: from neuromodulation to representation. Trends Cogn Sci, 2001, 5: 479-486

35 Li S C. Neurocomputational perspectives linking neuromodulation, processing noise, representational distinctiveness, and cognitive aging. In: Cabeza R, Nyberg L, Park D, eds. Cognitive Neuroscience of Aging: Linking Cognitive and Cerebral Aging. New York: Oxford University Press, 2005. 354-379

36 Voytek B, Kramer M A, Case J, et al. Age-related changes in 1/f neural electrophysiological noise. J Neurosci, 2015, 35: 13257-13265

37 Hasher L, Zacks R T. Working memory, comprehension and aging: a review and a new view. In: Bower G H, ed. The Psychology of Learning and Motivation. New York: Academic Press, 1988. 193-225

38 Hasher L, Zacks R T, May C P. Inhibitory control, circadian arousal, and age. In: Gopher D, Koriat A, eds. Attention and Performance XVIICognitive Regulation of Performance: Interaction of Theory and Application. Cambridge: MIT Press, 1999. 653-675

39 Hasher L, Lustig C, Zacks J M. Inhibitory mechanisms and the control of attention. In: Conway A, Jarrold C, Miyake A, eds. Variation in Working Memory. New York: Oxford University Press, 2008. 227-249 
40 Dempster F N. The rise and fall of the inhibitory mechanism: Toward a unified theory of cognitive development and aging. Dev Rev, 1992, 12: $45-75$

41 West R L. An application of prefrontal cortex function theory to cognitive aging. Psychol Bull, 1996, 120: 272-292

42 Grady C L. Cognitive neuroscience of aging. Ann New York Acad Sci, 2008, 1124: 127-144

43 Ferreira L K, Busatto G F. Resting-state functional connectivity in normal brain aging. Neurosci Biobehav Rev, 2013, 37: 384-400

44 Tatti E, Rossi S, Innocenti I, et al. Non-invasive brain stimulation of the aging brain: State of the art and future perspectives. Ageing Res Rev, 2016, 29: 66-89

45 Good C D, Johnsrude I S, Ashburner J, et al. A voxel-based morphometric study of ageing in 465 normal adult human brains. NeuroImage, 2001, 14: 21-36

46 Raz N, Lindenberger U, Rodrigue K M, et al. Regional brain changes in aging healthy adults: general trends, individual differences and modifiers. Cereb Cortex, 2005, 15: 1676-1689

47 Storsve A B, Fjell A M, Tamnes C K, et al. Differential longitudinal changes in cortical thickness, surface area and volume across the adult life span: regions of accelerating and decelerating change. J Neurosci, 2014, 34: 8488-8498

48 Groves A R, Smith S M, Fjell A M, et al. Benefits of multi-modal fusion analysis on a large-scale dataset: life-span patterns of inter-subject variability in cortical morphometry and white matter microstructure. NeuroImage, 2012, 63: 365-380

49 Yeatman J D, Wandell B A, Mezer A A. Lifespan maturation and degeneration of human brain white matter. Nat Commun, 2014, 5: 4932

50 Cardenas V A, Chao L L, Studholme C, et al. Brain atrophy associated with baseline and longitudinal measures of cognition. Neurobiol Aging, 2011, 32: 572-580

51 Madden D J, Bennett I J, Burzynska A, et al. Diffusion tensor imaging of cerebral white matter integrity in cognitive aging. Biochim Biophys Acta, 2012, 1822: 386-400

52 Rosen A C, Prull M W, Gabrieli J D E, et al. Differential associations between entorhinal and hippocampal volumes and memory performance in older adults. Behav Neurosci, 2003, 117: 1150-1160

53 Wolfson L, Wei X, Hall C B, et al. Accrual of MRI white matter abnormalities in elderly with normal and impaired mobility. J Neurol Sci, 2005, 232: $23-27$

54 Spreng R N, Wojtowicz M, Grady C L. Reliable differences in brain activity between young and old adults: a quantitative meta-analysis across multiple cognitive domains. Neurosci Biobehav Rev, 2010, 34: 1178-1194

55 Davis S W, Dennis N A, Daselaar S M, et al. Que PASA? The posterior-anterior shift in aging. Cereb Cortex, 2008, 18: 1201-1209

56 Cabeza R, Daselaar S M, Dolcos F, et al. Task-independent and task-specific age effects on brain activity during working memory, visual attention and episodic retrieval. Cereb Cortex, 2004, 14: 364-375

57 Dennis N A, Daselaar S, Cabeza R. Effects of aging on transient and sustained successful memory encoding activity. Neurobiol Aging, 2007, 28: $1749-1758$

58 Meulenbroek O, Petersson K M, Voermans N, et al. Age differences in neural correlates of route encoding and route recognition. NeuroImage, 2004, 22: 1503-1514

59 Cabeza R. Hemispheric asymmetry reduction in older adults: The HAROLD model. Psychol Aging, 2002, 17: 85-100

60 Reuter-Lorenz P A, Cappell K A. Neurocognitive aging and the compensation hypothesis. Curr Dir Psychol Sci, 2008, 17: 177-182

61 Park D C, Reuter-Lorenz P. The adaptive brain: aging and neurocognitive scaffolding. Annu Rev Psychol, 2009, 60: 173-196

62 Reuter-Lorenz P A, Park D C. How does it STAC up? Revisiting the scaffolding theory of aging and cognition. Neuropsychol Rev, 2014, 24: $355-370$

63 Lindenberger U, Baltes P B. Sensory functioning and intelligence in old age: a strong connection. Psychol Aging, 1994, 9: 339-355

64 Baltes P B, Lindenberger U. Emergence of a powerful connection between sensory and cognitive functions across the adult life span: a new window to the study of cognitive aging? Psychol Aging, 1997, 12: 12-21

65 Li S C, Lindenberger U, Hommel B, et al. Transformations in the couplings among intellectual abilities and constituent cognitive processes across the life span. Psychol Sci, 2004, 15: 155-163

66 Park J, Carp J, Hebrank A, et al. Neural specificity predicts fluid processing ability in older adults. J Neurosci, 2010, 30: 9253-9259

67 Dhanjal N S, Wise R J S. Frontoparietal cognitive control of verbal memory recall in Alzheimer's disease. Ann Neurol, 2014, 76: 241-251

68 Turner G R, Spreng R N. Prefrontal engagement and reduced default network suppression co-occur and are dynamically coupled in older adults: 
the default-executive coupling hypothesis of aging. J Cogn Neurosci, 2015, 27: 2462-2476

69 Spreng R N, Schacter D L. Default network modulation and large-scale network interactivity in healthy young and old adults. Cereb Cortex, 2012, 22: 2610-2621

70 Li H J, Hou X H, Liu H H, et al. Putting age-related task activation into large-scale brain networks: A meta-analysis of 114 fMRI studies on healthy aging. Neurosci Biobehav Rev, 2015, 57: 156-174

71 Li H J, Hou X H, Liu H H, et al. Toward systems neuroscience in mild cognitive impairment and Alzheimer's disease: A meta-analysis of 75 fMRI studies. Hum Brain Mapp, 2015, 36: 1217-1232

72 Biswal B B, Mennes M, Zuo X N, et al. Toward discovery science of human brain function. Proc Natl Acad Sci USA, 2010, 107: 4734-4739

73 Andrews-Hanna J R, Snyder A Z, Vincent J L, et al. Disruption of large-scale brain systems in advanced aging. Neuron, 2007, 56: 924-935

74 Tomasi D, Volkow N D. Functional connectivity hubs in the human brain. NeuroImage, 2011, 57: 908-917

75 Zhang H Y, Chen W X, Jiao Y, et al. Selective vulnerability related to aging in large-scale resting brain networks. PLoS ONE, 2014, 9: e108807

76 Geerligs L, Renken R J, Saliasi E, et al. A brain-wide study of age-related changes in functional connectivity. Cereb Cortex, 2015, 25: 19871999

77 Li R, Zhang S, Yin S, et al. The fronto-insular cortex causally mediates the default-mode and central-executive networks to contribute to individual cognitive performance in healthy elderly. Hum Brain Mapp, 2018, 39: 4302-4311

78 Zhang Y W, Zhao Z L, Qi Z, et al. Local-to-remote cortical connectivity in amnestic mild cognitive impairment. Neurobiol Aging, 2017, 56: 138-149

79 Wang Z, Qiao K, Chen G, et al. Functional connectivity changes across the spectrum of subjective cognitive decline, amnestic mild cognitive impairment and Alzheimer's disease. Front Neuroinform, 2019, 13: 26

80 Campbell K L, Schacter D L. Ageing and the resting state: is cognition obsolete? Lang Cogn Neurosci, 2017, 32: 661-668

81 Geerligs L, Tsvetanov K A. The use of resting state data in an integrative approach to studying neurocognitive ageing-commentary on Campbell and Schacter (2016). Lang Cogn Neurosci, 2017, 32: 684-691

82 Bookheimer S Y, Salat D H, Terpstra M, et al. The Lifespan Human Connectome Project in Aging: an overview. NeuroImage, 2019, 185: 335348

83 Wierenga C E, Hays C C, Zlatar Z Z. Cerebral blood flow measured by arterial spin labeling MRI as a preclinical marker of Alzheimer's disease. J Alzheimers Dis, 2014, 42: S411-S419

84 Hays C C, Zlatar Z Z, Wierenga C E. The utility of cerebral blood flow as a biomarker of preclinical Alzheimer's disease. Cell Mol Neurobiol, 2016, 36: 167-179

85 Zhang N, Gordon M L, Goldberg T E. Cerebral blood flow measured by arterial spin labeling MRI at resting state in normal aging and Alzheimer's disease. Neurosci Biobehav Rev, 2017, 72: 168-175

86 Catchlove S J, Macpherson H, Hughes M E, et al. An investigation of cerebral oxygen utilization, blood flow and cognition in healthy aging. PLoS ONE, 2018, 13: e0197055

$87 \mathrm{Lu} \mathrm{H}, \mathrm{Xu} \mathrm{F}$, Rodrigue K M, et al. Alterations in cerebral metabolic rate and blood supply across the adult lifespan. Cereb Cortex, 2011, 21: $1426-1434$

88 Chiacchiaretta P, Cerritelli F, Bubbico G, et al. Reduced dynamic coupling between spontaneous BOLD-CBF fluctuations in older adults: a dual-echo pCASL study. Front Aging Neurosci, 2018, 10: 115

89 Heo S, Prakash R S, Voss M W, et al. Resting hippocampal blood flow, spatial memory and aging. Brain Res, 2010, 1315: 119-127

90 Mak H K F, Chan Q, Zhang Z, et al. Quantitative assessment of cerebral hemodynamic parameters by QUASAR arterial spin labeling in Alzheimer's disease and cognitively normal elderly adults at 3-Tesla. J Alzheimers Dis, 2012, 31: 33-44

91 Staffaroni A M, Cobigo Y, Elahi F M, et al. A longitudinal characterization of perfusion in the aging brain and associations with cognition and neural structure. Hum Brain Mapp, 2019, 40: 3522-3533

92 De Vis J B, Hendrikse J, Bhogal A, et al. Age-related changes in brain hemodynamics; A calibrated MRI study. Hum Brain Mapp, 2015, 36: 3973-3987

93 Sofi F, Valecchi D, Bacci D, et al. Physical activity and risk of cognitive decline: a meta-analysis of prospective studies. J Intern Med, 2011, 269: $107-117$

94 Daskalopoulou C, Stubbs B, Kralj C, et al. Physical activity and healthy ageing: A systematic review and meta-analysis of longitudinal cohort 
studies. Ageing Res Rev, 2017, 38: 6-17

95 Colcombe S J, Erickson K I, Scalf P E, et al. Aerobic exercise training increases brain volume in aging humans. J Gerontol A Biol Sci Med Sci, 2006, 61: 1166-1170

96 Best J R, Chiu B K, Liang Hsu C, et al. Long-term effects of resistance exercise training on cognition and brain volume in older women: results from a randomized controlled trial. J Int Neuropsychol Soc, 2015, 21: 745-756

97 Erickson K I, Voss M W, Shaurya Prakash R, et al. Exercise training increases size of hippocampus and improves memory. Proc Natl Acad Sci USA, 2011, 108: 3017-3022

98 Erickson K I, Raji C A, Lopez O L, et al. Physical activity predicts gray matter volume in late adulthood: The Cardiovascular Health Study. Neurology, 2010, 75: 1415-1422

99 Voss M W, Heo S, Prakash R S, et al. The influence of aerobic fitness on cerebral white matter integrity and cognitive function in older adults: results of a one-year exercise intervention. Hum Brain Mapp, 2013, 34: 2972-2985

100 Liu J, Tao J, Liu W, et al. Different modulation effects of Tai Chi Chuan and Baduanjin on resting-state functional connectivity of the default mode network in older adults. Soc Cogne Affect Neurosci, 2019, 14: 217-224

101 Voss M W, Prakash R S, Erickson K I, et al. Plasticity of brain networks in a randomized intervention trial of exercise training in older adults. Fronti Aging Neurosci, 2010, 2: 32

102 Prehn K, Lesemann A, Krey G, et al. Using resting-state fMRI to assess the effect of aerobic exercise on functional connectivity of the DLPFC in older overweight adults. Brain Cogn, 2019, 131: 34-44

103 Wu M T, Tang P F, Goh J O S, et al. Task-switching performance improvements after Tai Chi Chuan training are associated with greater prefrontal activation in older adults. Front Aging Neurosci, 2018, 10: 280

104 Tao J, Chen X, Liu J, et al. Tai Chi Chuan and Baduanjin mind-body training changes resting-state low-frequency fluctuations in the frontal lobe of older adults: a resting-state fMRI study. Front Hum Neurosci, 2017, 11: 514

105 Tao J, Chen X, Egorova N, et al. Tai Chi Chuan and Baduanjin practice modulates functional connectivity of the cognitive control network in older adults. Sci Rep, 2017, 7: 41581

106 Tarumi T, Ayaz Khan M, Liu J, et al. Cerebral hemodynamics in normal aging: central artery stiffness, wave reflection, and pressure pulsatility. J Cereb Blood Flow Metab, 2014, 34: 971-978

107 Burdette J H, Laurienti P J, Espeland M A, et al. Using network science to evaluate exercise-associated brain changes in older adults. Front Aging Neurosci, 2010, 2: 23

108 Chapman S B, Aslan S, Spence J S, et al. Shorter term aerobic exercise improves brain, cognition, and cardiovascular fitness in aging. Front Aging Neurosci, 2013, 5: 75

109 Thomas B P, Yezhuvath U S, Tseng B Y, et al. Life-long aerobic exercise preserved baseline cerebral blood flow but reduced vascular reactivity to $\mathrm{CO}_{2}$. J Magn Reson Imag, 2013, 38: 1177-1183

110 Picorelli A M A, Pereira L S M, Pereira D S, et al. Adherence to exercise programs for older people is influenced by program characteristics and personal factors: a systematic review. J Physiother, 2014, 60: 151-156

111 Sprague B N, Freed S A, Webb C E, et al. The impact of behavioral interventions on cognitive function in healthy older adults: A systematic review. Ageing Res Rev, 2019, 52: 32-52

112 Graves L E F, Ridgers N D, Williams K, et al. The physiological cost and enjoyment of Wii Fit in adolescents, young adults, and older adults. J Phys Act Health, 2010, 7: 393-401

113 Studenski S, Perera S, Hile E, et al. Interactive video dance games for healthy older adults. J Nutr Health Aging, 2010, 14: 850-852

114 Singh D K A, Rajaratnam B S, Palaniswamy V, et al. Effects of balance-focused interactive games compared to therapeutic balance classes for older women. Climacteric, 2013, 16: 141-146

115 Nicholson V P, McKean M, Lowe J, et al. Six weeks of unsupervised Nintendo Wii Fit gaming is effective at improving balance in independent older adults. J Aging Phys Act, 2015, 23: 153-158

116 Maillot P, Perrot A, Hartley A. Effects of interactive physical-activity video-game training on physical and cognitive function in older adults. Psychol Aging, 2012, 27: 589-600

117 Stanmore E, Stubbs B, Vancampfort D, et al. The effect of active video games on cognitive functioning in clinical and non-clinical populations: A meta-analysis of randomized controlled trials. Neurosci Biobehav Rev, 2017, 78: 34-43 
118 Li H, Li J, Li N, et al. Cognitive intervention for persons with mild cognitive impairment: A meta-analysis. Ageing Res Rev, 2011, 10: 285-296

119 Cognitive Training China Expert Consensus Writing Group, Cognitive Disorders Professional Committee Of Chinese Medical Doctor Association Neurologist Branch. Cognitive training China expert consensus (in Chinese). Natl Med J China, 2019, 99: 4-8 [认知训练中国专家 共识写作组, 中国医师协会神经内科医师分会认知障碍疾病专业委员会. 认知训练中国专家共识. 中华医学杂志, 2019, 99: 4-8]

120 Han X, Shi D Q, Zhou X W, et al. The training and transfer effect of cognitive training in old adults (in Chinese). Adv Psychol Sci, 2016, 24: 909-922 [韩笑, 石岱青, 周晓文, 等. 认知训练对健康老年人认知能力的影响. 心理科学进展, 2016, 24: 909-922]

121 Schaie K W, Willis S L. The seattle longitudinal study of adult cognitive development. ISSBD Bull, 2010, 57: 24-29

122 Ball K, Berch D B, Helmers K F, et al. Effects of cognitive training interventions with older adults. JAMA, 2002, 288: 2271-2281

123 Chan M Y, Haber S, Drew L M, et al. Training older adults to use tablet computers: does it enhance cognitive function? Gerontologist, 2016, 56: $475-484$

124 Park D C, Lodi-Smith J, Drew L, et al. The impact of sustained engagement on cognitive function in older adults: The Synapse Project. Psychol Sci, 2014, 25: 103-112

$125 \mathrm{Du} \mathrm{X}$, Ji Y, Chen T, et al. Can working memory capacity be expanded by boosting working memory updating efficiency in older adults? Psychol Aging, 2018, 33: 1134-1151

126 Heinzel S, Lorenz R C, Brockhaus W R, et al. Working memory load-dependent brain response predicts behavioral training gains in older adults. J Neurosci, 2014, 34: 1224-1233

127 Heinzel S, Lorenz R C, Pelz P, et al. Neural correlates of training and transfer effects in working memory in older adults. NeuroImage, 2016, 134: $236-249$

128 Wenger E, Schaefer S, Noack H, et al. Cortical thickness changes following spatial navigation training in adulthood and aging. NeuroImage, 2012, 59: 3389-3397

129 Mozolic J L, Hayasaka S, Laurienti P J. A cognitive training intervention increases resting cerebral blood flow in healthy older adults. Front Hum Neurosci, 2010, 4: 16

130 Chapman S B, Spence J S, Aslan S, et al. Enhancing innovation and underlying neural mechanisms via cognitive training in healthy older adults. Front Aging Neurosci, 2017, 9: 314

131 Powers K L, Brooks P J, Aldrich N J, et al. Effects of video-game play on information processing: a meta-analytic investigation. Psychon Bull Rev, 2013, 20: 1055-1079

132 Toril P, Reales J M, Ballesteros S. Video game training enhances cognition of older adults: a meta-analytic study. Psychol Aging, 2014, 29: 706716

133 Wang P, Liu H H, Zhu X T, et al. Action video game training for healthy adults: a meta-analytic study. Front Psychol, 2016, 7: 907

134 Toril P, Reales J M, Mayas J, et al. Video game training enhances visuospatial working memory and episodic memory in older adults. Front Hum Neurosci, 2016, 10: 206

135 Kühn S, Romanowski A, Schilling C, et al. The neural basis of video gaming. Transl Psychiatry, 2011, 1: e53

136 Kühn S, Gallinat J. Amount of lifetime video gaming is positively associated with entorhinal, hippocampal and occipital volume. Mol Psychiatry, 2014, 19: 842-847

137 Wang P, Zhu X T, Qi Z, et al. Neural basis of enhanced executive function in older video game players: an fMRI study. Front Aging Neurosci, 2017, 9: 382

138 Hou H Y, Jia X Z, Wang P, et al. Intrinsic resting-state activity in older adults with video game experience. Front Aging Neurosci, 2019, 11: 119

139 Perani D, Abutalebi J. Bilingualism, dementia, cognitive and neural reserve. Curr Opin Neurol, 2015, 28: 618-625

140 Wang H X, Karp A, Winblad B, et al. Late-life engagement in social and leisure activities is associated with a decreased risk of dementia: a longitudinal study from the Kungsholmen project. Am J Epidemiol, 2002, 155: 1081-1087

141 Crowe M, Andel R, Pedersen N L, et al. Does participation in leisure activities lead to reduced risk of Alzheimer's disease? A prospective study of Swedish twins. J Gerontol B Psychol Sci Soc Sci, 2003, 58: P249-P255

142 Verghese J, Lipton R B, Katz M J, et al. Leisure activities and the risk of dementia in the elderly. N Engl J Med, 2003, 348: 2508-2516

143 Scarmeas N, Levy G, Tang M X, et al. Influence of leisure activity on the incidence of Alzheimer's Disease. Neurology, 2001, 57: 2236-2242

144 Festini S B, McDonough I M, Park D C. The busier the better: greater busyness is associated with better cognition. Front Aging Neurosci, 2016, 8: 98 
145 Li X, Ma C, Zhang J, et al. Prevalence of and potential risk factors for mild cognitive impairment in community-dwelling residents of Beijing. J Am Geriatr Soc, 2013, 61: 2111-2119

146 Paggi M E, Jopp D, Hertzog C. The importance of leisure activities in the relationship between physical health and well-being in a life span sample. Gerontology, 2016, 62: 450-458

147 Gow A J, Pattie A, Deary I J. Lifecourse activity participation from early, mid, and later adulthood as determinants of cognitive aging: the lothian birth cohort 1921. J Gerontol B Psychol Sci Soc Sci, 2017, 72: 25-37

148 Blumenthal J A, Smith P J, Mabe S, et al. Lifestyle and neurocognition in older adults with cardiovascular risk factors and cognitive impairment. Psychosom Med, 2017, 79: 719-727

149 Blumenthal J A, Smith P J, Mabe S, et al. Lifestyle and neurocognition in older adults with cognitive impairments. Neurology, 2019, 92: e212e223

150 Lehtisalo J, Ngandu T, Valve P, et al. Nutrient intake and dietary changes during a 2-year multi-domain lifestyle intervention among older adults: secondary analysis of the Finnish Geriatric Intervention Study to Prevent Cognitive Impairment and Disability (FINGER) randomised controlled trial. Br J Nutr, 2017, 118: 291-302

151 Lam L C W, Chan W C, Leung T, et al. Would older adults with mild cognitive impairment adhere to and benefit from a structured lifestyle activity intervention to enhance cognition?: a cluster randomized controlled trial. PLoS ONE, 2015, 10: e0118173

152 Clark F, Jackson J, Carlson M, et al. Effectiveness of a lifestyle intervention in promoting the well-being of independently living older people: results of the Well Elderly 2 Randomised Controlled Trial. J Epidemiol Community Health, 2012, 66: 782-790

153 Annweiler C, Annweiler T, Montero-Odasso M, et al. Vitamin D and brain volumetric changes: Systematic review and meta-analysis. Maturitas, 2014, 78: 30-39

154 Berti V, Murray J, Davies M, et al. Nutrient patterns and brain biomarkers of Alzheimer's disease in cognitively normal individuals. J Nutr Health Aging, 2015, 19: 413-423

155 Zwilling C E, Talukdar T, Zamroziewicz M K, et al. Nutrient biomarker patterns, cognitive function, and fMRI measures of network efficiency in the aging brain. NeuroImage, 2019, 188: 239-251

156 Mosconi L, Murray J, Davies M, et al. Nutrient intake and brain biomarkers of Alzheimer's disease in at-risk cognitively normal individuals: a cross-sectional neuroimaging pilot study. BMJ Open, 2014, 4: e004850

157 Walters M J, Sterling J, Quinn C, et al. Associations of lifestyle and vascular risk factors with Alzheimer's brain biomarker changes during middle age: a 3-year longitudinal study in the broader New York City area. BMJ Open, 2018, 8: e023664

158 Cabeza R, Nyberg L, Park D C. Cognitive Neuroscience of Aging. New York: Oxford University Press, 2006

159 Eyler L T, Sherzai A, Kaup A R, et al. A review of functional brain imaging correlates of successful cognitive aging. Biol Psychiatry, 2011, 70: $115-122$

160 Damoiseaux J S, Beckmann C F, Arigita E J S, et al. Reduced resting-state brain activity in the "default network" in normal aging. Cereb Cortex, 2008, 18: 1856-1864

161 Sambataro F, Murty V P, Callicott J H, et al. Age-related alterations in default mode network: impact on working memory performance. Neurobiol Aging, 2010, 31: 839-852

162 Onoda K, Ishihara M, Yamaguchi S. Decreased functional connectivity by aging is associated with cognitive decline. J Cogn Neurosci, 2012, 24: 2186-2198

163 Craik F I M. On the transfer of information from temporary to permanent memory. Phil Trans R Soc Lond B, 1983, 302: 341-359

164 Craik F I M, Jennings J M. Human memory. In: Craik F I M, Salthouse T A, eds. Handbook of Aging and Cognition. Hillsdale: Erlbaun. 1992. 51-110

165 Baltes P B, Baltes M M. Psychological perspectives on successful aging: the model of selective optimization with compensation. In: Baltes P B, Baltes M M, eds. Successful Aging: Perspectives From the Behavioral Sciences. Cambridge: Cambridge University Press, 1990. 1-34

166 Park H, Kennedy K M, Rodrigue K M, et al. An fMRI study of episodic encoding across the lifespan: changes in subsequent memory effects are evident by middle-age. Neuropsychologia, 2013, 51: 448-456

167 Kennedy K M, Rodrigue K M, Bischof G N, et al. Age trajectories of functional activation under conditions of low and high processing demands: An adult lifespan fMRI study of the aging brain. NeuroImage, 2015, 104: 21-34

168 Rovio S, Spulber G, Nieminen L J, et al. The effect of midlife physical activity on structural brain changes in the elderly. Neurobiol Aging, 
2010, 31: 1927-1936

169 Grady C L, Springer M V, Hongwanishkul D, et al. Age-related changes in brain activity across the adult lifespan. J Cogn Neurosci, 2006, 18: $227-241$

170 Zuo X N, Kelly C, Di Martino A, et al. Growing together and growing apart: regional and sex differences in the lifespan developmental trajectories of functional homotopy. J Neurosci, 2010, 30: 15034-15043

171 Chan M Y, Park D C, Savalia N K, et al. Decreased segregation of brain systems across the healthy adult lifespan. Proc Natl Acad Sci USA, 2014, 111: E4997-E5006

172 Betzel R F, Byrge L, He Y, et al. Changes in structural and functional connectivity among resting-state networks across the human lifespan. NeuroImage, 2014, 102: 345-357

173 Zuo X N, He Y, Betzel R F, et al. Human connectomics across the life span. Trends Cogn Sci, 2017, 21: 32-45

174 Potvin O, Mouiha A, Dieumegarde L, et al. Normative data for subcortical regional volumes over the lifetime of the adult human brain. NeuroImage, 2016, 137: 9-20

175 Coupé P, Manjón J V, Lanuza E, et al. Lifespan changes of the human brain in Alzheimer's disease. Sci Rep, 2019, 9: 3998

176 Fotenos A F, Snyder A Z, Girton L E, et al. Normative estimates of cross-sectional and longitudinal brain volume decline in aging and AD. Neurology, 2005, 64: 1032-1039

177 Bertolero M A, Yeo B T T, D’Esposito M. The modular and integrative functional architecture of the human brain. Proc Natl Acad Sci USA, 2015, 112: E6798-E6807

178 Bassett D S, Wymbs N F, Porter M A, et al. Dynamic reconfiguration of human brain networks during learning. Proc Natl Acad Sci USA, 2011, 108: $7641-7646$

179 Bullmore E, Sporns O. The economy of brain network organization. Nat Rev Neurosci, 2012, 13: 336-349

180 Sporns O. Contributions and challenges for network models in cognitive neuroscience. Nat Neurosci, 2014, 17: 652-660

181 Cole M W, Reynolds J R, Power J D, et al. Multi-task connectivity reveals flexible hubs for adaptive task control. Nat Neurosci, 2013, 16: $1348-1355$

182 Crowell C A, Davis S W, Beynel L, et al. Older adults benefit from more widespread brain network integration during working memory. NeuroImage, 2020, 218: 116959

183 Han B X. New direction of cognitive and brain aging: The importance of human brain frontoparietal network study (in Chinese). Chin Sci Bull, 2015, 60: 3180-3182 [韩布新. 认知与脑老化研究的新方向: 论人脑额顶网络研究的重要性. 科学通报, 2015, 60: 3180-3182]

184 Braver T S, Barch D M, Keys B A, et al. Context processing in older adults: evidence for a theory relating cognitive control to neurobiology in healthy aging. J Exp Psychol Gen, 2001, 130: 746-763

185 Braver T S, Barch D M. A theory of cognitive control, aging cognition, and neuromodulation. Neurosci Biobehav Rev, 2002, 26: 809-817

186 Bäckman L, Nyberg L, Soveri A, et al. Effects of working-memory training on striatal dopamine release. Science, 2011, 333: 718

187 Bäckman L, Karlsson S, Fischer H, et al. Dopamine $\mathrm{D}_{1}$ receptors and age differences in brain activation during working memory. Neurobiol Aging, 2011, 32: 1849-1856

188 Braskie M N, Wilcox C E, Landau S M, et al. Relationship of striatal dopamine synthesis capacity to age and cognition. J Neurosci, 2008, 28: 14320-14328

189 Zanto T P, Gazzaley A. Chapter 20-Aging of the frontal lobe. In: D’Esposito M, Grafman J H, eds. Handbook of Clinical Neurology. Amsterdam: Elsevier Ltd, 2019. 369-389

190 Zinke K, Zeintl M, Rose N S, et al. Working memory training and transfer in older adults: effects of age, baseline performance, and training gains. Dev Psychol, 2014, 50: 304-315

191 Zhu Z D, Duan Y X, Wang S P. The baseline fluid intelligence modulated the transfer effect from working memory to fluid intelligence (in Chinese). Acta Psychol Sin, 2017, 49: 1022-1030 [朱祖德, 段懿行, 王穗苹. 个体差异对工作记忆训练迁移效果的调节. 心理学报, 2017, 49: 1022-1030]

192 Gallen C L, Turner G R, Adnan A, et al. Reconfiguration of brain network architecture to support executive control in aging. Neurobiol Aging, 2016, 44: 42-52

193 Liao X, Vasilakos A V, He Y. Small-world human brain networks: Perspectives and challenges. Neurosci Biobehav Rev, 2017, 77: 286-300

194 Reynolds C A, Finkel D. A meta-analysis of heritability of cognitive aging: minding the "missing heritability" gap. Neuropsychol Rev, 2015, 
25: $97-112$

195 Papenberg G, Salami A, Persson J, et al. Genetics and functional imaging: effects of $A P O E, B D N F$, COMT, and KIBRA in aging. Neuropsychol Rev, 2015, 25: 47-62

196 Laukka E J, Lövdén M, Herlitz A, et al. Genetic effects on old-age cognitive functioning: a population-based study. Psychol Aging, 2013, 28: $262-274$

197 Corder E H, Saunders A M, Strittmatter W J, et al. Gene dose of apolipoprotein E type 4 allele and the risk of Alzheimer's disease in late onset families. Science, 1993, 261: 921-923

198 Li R, Wan W, Li J. KIBRA polymorphism modulates gray matter volume to influence cognitive ability in the elderly. Brain Imag Behav, 2020, 14: $1388-1394$

199 Chen Y, Chen K, Zhang J, et al. Disrupted functional and structural networks in cognitively normal elderly subjects with the $A P O E 4$ allele. Neuropsychopharmacology, 2015, 40: 1181-1191

200 Ma C, Wang J, Zhang J, et al. Disrupted brain structural connectivity: pathological interactions between genetic APOE $\varepsilon 4$ status and developed MCI condition. Mol Neurobiol, 2017, 54: 6999-7007

201 Bondi M W, Houston W S, Eyler L T, et al. fMRI evidence of compensatory mechanisms in older adults at genetic risk for Alzheimer disease. Neurology, 2005, 64: 501-508

202 Bian Y Y, Zhang Y J, Liu P P, et al. The regional and disciplinary imbalance of geriatric psychology research in China (in Chinese). Chin J Gerontol, 2017, 37: 2525-2528 [边玉芗, 张玉静, 刘萍萍, 等. 中国老年心理学研究的地区与学科不均衡. 中国老年学杂志, 2017, 37: 25252528]

203 Chen Y J, Xu K, Yang C S, et al. Beijing Aging Brain Rejuvenation Initiative: aging with grace (in Chinese). Sci Sin Vitae, 2018, 48: 721-734 [陈姚静, 徐凯, 杨财水, 等. 优雅地老去——北京BABRI老年脑健康计划. 中国科学: 生命科学, 2018, 48: 721-734]

204 Anderson N D, Craik F I M. 50 years of cognitive aging theory. J Gerontol B Psychol Sci Soc Sci, 2017, 72: 1-6

205 Coffman B A, Clark V P, Parasuraman R. Battery powered thought: enhancement of attention, learning, and memory in healthy adults using transcranial direct current stimulation. Neurolmage, 2014, 85: 895-908

206 Mishra J, Anguera J A, Gazzaley A. Video games for neuro-cognitive optimization. Neuron, 2016, 90: 214-218

207 Li J, Wang L M. Study on income conditions of the elderly in China —analysis based on data from the fourth sample survey on the living conditions of China's urban and rural older persons (in Chinese). Sci Res Aging, 2018, 6:3-17 [李军, 王丽民. 我国老年人的收入状况一基于 第四次中国城乡老年人生活状况抽样调查数据的分析. 老龄科学研究, 2018, 6: 3-17]

208 Leon M, Woo C. Environmental enrichment and successful aging. Front Behav Neurosci, 2018, 12: 155

209 Hertzog C, Kramer A F, Wilson R S, et al. Enrichment effects on adult cognitive development. Psychol Sci Public Interest, 2008, 9: 1-65

210 Patel T R. Environmental enrichment: aging and memory. Yale J Biol Med, 2012, 85: 491-500

211 Jia J, Wang F, Wei C, et al. The prevalence of dementia in urban and rural areas of China. Alzheimers Dement, 2014, 10: 1-9

212 Xie H. Construction of active aging model: an empirical study based on WHO's framework for active aging policy (in Chinese). Dissertation for Doctoral Degree. Jinan: Shandong University, 2019 [谢轫. 积极老龄化模型构建: 基于世界卫生组织积极老龄化框架的实证研究. 博士学位 论文. 济南: 山东大学, 2019]

213 Zheng J K, Hu R R, Hong Z J, et al. Relationship between cognitive abiity and negative emotion of urban and rural elderly (in Chinese). Chin J Health Psychol, 2020, 28: 142-145 [郑俊㑆, 胡蓉蓉, 洪紫静, 等. 城乡老年人认知能力与负性情绪的相关性. 中国健康心理学杂志, 2020, 28: 142-145]

214 Wang Z, Chen Z, Zhang L, et al. Status of hypertension in China. Circulation, 2018, 137: 2344-2356

215 Wang L, Gao P, Zhang M, et al. Prevalence and ethnic pattern of diabetes and prediabetes in China in 2013. JAMA, 2017, $317: 2515$

216 Wang F, Zhang Q E, Zhang L, et al. Prevalence of major depressive disorder in older adults in China: A systematic review and meta-analysis. J Affect Disord, 2018, 241: 297-304

217 Jia J, Zhou A, Wei C, et al. The prevalence of mild cognitive impairment and its etiological subtypes in elderly Chinese. Alzheimers Dement, 2014, 10: 439-447

218 Arvanitakis Z, Wilson R S, Bienias J L, et al. Diabetes mellitus and risk of Alzheimer disease and decline in cognitive function. Arch Neurol, 2004, 61: 661-666

219 Gifford K A, Badaracco M, Liu D, et al. Blood pressure and cognition among older adults: a meta-analysis. Arch Clin Neuropsychol, 2013, 28: 
649-664

220 Shimada H, Park H, Makizako H, et al. Depressive symptoms and cognitive performance in older adults. J Psychiatr Res, 2014, 57: 149-156

221 Kato N, Takeuchi F, Tabara Y, et al. Meta-analysis of genome-wide association studies identifies common variants associated with blood pressure variation in east Asians. Nat Genet, 2011, 43: 531-538

222 Cardio-Cerebral-Vascular Branch Hypertension Group of The Chinese Medical Association. Salt-limiting management to control hypertension expert guidance in China (in Chinese). Chin J Hypertens, 2015, 23: 1028-1034 [中华医学会心血管病学分会高血压学组. 限盐管理控制高血. 压中国专家指导意见. 中华高血压杂志, 2015, 23: 1028-1034]

223 Joint Committee for Guideline Revision. 2018 Chinese Guidelines for Prevention and Treatment of Hypertension-A report of the Revision Committee of Chinese Guidelines for Prevention and Treatment of Hypertension. J Geriatr Cardiol, 2019, 16: 182-241

\title{
Cognitive neuroscience of aging: present research status and future prospect
}

\author{
LIU HanHui ${ }^{1} \&$ LI HuiJie ${ }^{2,3}$ \\ 1 Department of Youth Work, Chinese Youth University for Political Sciences, Beijing 100089, China; \\ 2 CAS Key Laboratory of Behavioral Science, Institute of Psychology, Chinese Academy of Sciences, Beijing 100101, China; \\ 3 Department of Psychology, University of Chinese Academy of Sciences, Beijing 100049, China
}

Population aging has become a serious social problem for China and the world. Researchers have focused on the study of cognitive neuroscience in aging, revealing the underlying mechanisms and laws of brain aging from cognition, brain structure and brain function, exploring and developing effective intervention approaches and tools to delay cognitive decline. It is of great significance to maintain the brain health and improve quality of life. In this manuscript, we overviewed the neural mechanisms of cognitive aging according to the evidence of brain structure aging, brain function aging, cerebral blood flow and brain aging. Multiple cognitive aging theories and hypotheses have been constructed from the perspective of compensation and dedifferentiation, however, these theories and hypotheses take less account of findings from studies on brain structure aging, resting-state neuroimaging as well as brain blood flow. Adhering to regular exercise, engaging in more cognitive activities and maintaining a healthy lifestyle and diet pattern could help older adults improve cognitive function and delay cognitive decline. We predicted the future directions of cognitive neuroscience of aging from development of cognitive aging theory, studies on cognitive aging from the perspective of lifespan development, functional networks of aging brain and brain plasticity, and the effect of gene on brain aging. Finally, we discussed the future prospect focusing on the construction of the behavior-gene-brain database of adult lifespan development in China, using and developing new techniques to study cognitive aging and explore effective brain function improvement training methods, and conducting studies on rural older adults and clinical older populations.

older adults, aging, prefrontal lobe, intervention, neuroscience

doi: $10.1360 /$ SSV-2020-0256 